\title{
Modeling, Stability and Control of Biped Robots A General Framework
}

Yildirim Hurmuzlu — Frank Génot — Bernard Brogliato

$$
\mathbf{N}^{\circ} 4290
$$

Octobre 2001

THÈME 4 



\title{
Modeling, Stability and Control of Biped Robots A General Framework
}

\author{
Yildirim Hurmuzlu*, Frank Génot ${ }^{\dagger}$, Bernard Brogliato ${ }^{\ddagger}$ \\ Thème 4 - Simulation et optimisation \\ de systèmes complexes \\ Projet BIP
}

Rapport de recherche $\mathrm{n}^{\circ} 4290$ - Octobre 2001 - 88 pages

\begin{abstract}
This paper seeks to review the developments in the field within the framework of stability and control of systems subject to unilateral constraints. Particular emphasis is given to three main issues that, in our view, form the underlying theory in the study of such systems. Impact of the lower limbs with the walking surface and its effect on the walking dynamics is considered first. Several key issues such as multiple impacts, restitution and friction laws, and existence and uniqueness of solutions are reviewed in detail. Next, we consider the dynamic stability of bipedal gait. We review the use of discrete maps in studying the stability of the closed orbits that represent the dynamics of a biped, which can be characterized as a hybrid system. Last, we consider the control schemes that have been used in regulating the motion of bipedal systems, and we seek to identify the needed future developments.
\end{abstract}

Key-words: Biped robots; nonsmooth mechanics; multiple impact laws; hybrid system; gait stability; control synthesis.

* Mechanical Engineering Department, Southern Methodist University, Dallas, TX 75252, USA. Email: hurmuzlu@seas.smu.edu

${ }^{\dagger}$ INRIA Rocquencourt, Projet MACS, Domaine de Voluceau - BP 105, 78153 Le Chesnay Cedex, France. Email: Frank.Genot@inria.fr

‡ INRIA Rhône-Alpes, Projet BIP, ZIRST, 655 avenue de l'Europe, Montbonnot, 38334

St Ismier Cedex, France. Email: Bernard.Brogliato@inrialpes.fr 


\section{Modélisation, stabilité et contrôle des robots bipèdes - Un cadre général}

Résumé : Cet article porte sur la modélisation et le contrôle de systèmes de locomotion à deux pattes. Plus précisemment, il propose un état de l'art des développements récents dans ce domaine sous l'angle de la stabilité et du contrôle des systèmes avec contraintes unilatérales. L'accent est mis sur trois problèmes fondamentaux qui constituent, de notre point de vue, un cadre théorique complet pour l'étude de ces systèmes de locomotion. Dans un premier temps, nous abordons le phénomène d'impact des membres inférieurs avec la surface d'appui ainsi que son effet sur la dynamique. Plusieurs points clé tels que les impacts multiples, les lois de restitution et de frottement sec ainsi que les problèmes d'existence et d'unicité de la trajectoires sont présentés dans les détails. La seconde partie est consacrée à l'étude de la stabilité dynamique de la marche. En montrant que ces systèmes mécaniques constituent une classe particulière de systèmes hybrides, nous proposons l'utilisation des sections de Poincaré pour l'étude de la stabilité des trajectoires closes. Finalement, différents schémas de contrôle, proposés dans la littérature, pour réguler la marche des robots bipèdes sont passés en revue sous l'angle de cette modélisation. Nous concluons en tentant d'identifier de futurs développements nécessaires à ce domaine.

Mots-clés : Robots bipèdes; mécanique non-lisse; lois de chocs multiples; systèmes hybrides; stabilité de la marche; synthèse de contrôleurs. 


\section{Introduction}

In general, a bipedal locomotion system consists of several members that are interconnected with actuated joints. In essence, a man-made walking robot is nothing more than a robotic manipulator with a detachable and moving base. Design of bipedal robots has been largely influenced by the most sophisticated and versatile biped known to man, the man himself. Therefore, most of the models/machines developed bear a strong resemblance to the human body. Almost any model or machine can be characterized as having two lower limbs that are connected through a central member. Although the complexity of system depends on the number of degrees of freedom, the existence of feet structures, upper limbs etc., it is widely known that even extremely simple unactuated systems can generate ambulatory motion. A bipedal locomotion system can have a very simple structure with three point masses connected with massless links (Garcia, Chatterjee, Ruina \& Coleman (1997)) or very complex structure that mimics the human body (Vukobratovic, Borovac, Surla \& Stokic (1990)). In both cases the system can achieve stable locomotion. The robotics community has been involved in the field of modeling and control of bipeds for many years. The books by Vukobratovic (Vukobratovic (1976); Vukobratovic, Borovac, Surla \& Stokic (1990)), Raibert (Raibert (1986)) and Todd (Todd (1985)) are worth reading as an introduction to the field. Recently, the interest for bipedal locomotion has been reinforced by the construction by Honda of a collection of robots whose behavior as a humanoid is quite impressive, as videos witness. The interested reader should refer to the following web pages to get an overview of the community of researchers working on biped robots:

http://robby.caltech.edu/ kajita/bipedsite.html http://www.fzi.de/divisions/ipt/WMC/preface/preface.html

Nevertheless, and despite the technological exploit achieved by Honda's engineers (Japan is certainly the country where bipedal locomotion has received the most attention and has the longest history), some fundamental modeling and control problems have still not been addressed nor solved in the related literature. One may notice in particular that the locomotion of Honda's P3 prototype remains far from classical human walking patterns at the same speeds. Although Honda (HONDA) did not publish many details

$\mathrm{RR} \mathrm{n}^{\circ} 4290$ 
neither on the mechanical part nor on the implemented control heuristic, it is easy to see on the available videos that P3's foot strike is anomalous and leads to some transient instability (http://www.honda-p3.com). The number of foot design patents taken out by Honda (up to an air-bag like planter arch) reveals again that foot-ground impact remains one of the main difficulties one has to face in the design of robust control laws for walking robots and will become the key issue with increasing horizontal velocity requirement. This problem, however, is more sensitive for two-legged robots than for multi-legged ones due to the almost straight leg configuration and the bigger load at impact time for the former, leading to stronger velocity jumps of the center of mass. While Honda's engineers seem to consider these velocity jumps as unwanted perturbations and thus appeal to mechanical astutenesses to smooth the trajectory, we argue that impact is an intrinsic feature of mechanical systems like biped robots and should be taken as such in the controller design. Other bipedal robots have been designed. Among the most advanced projects, lwe cite the Waseda University Humanoid Robotics Institute biped (http://www.humanoid.rise.waseda.ac.jp/booklet/kato_4.html), the MIT Leg Laboratory robots (http://www.ai.mit.edu/projects/leglab/robots/robots.html) and the LMS-INRIA BIP system (http://www.inrialpes.fr/bip). Other projects can be found at http://www.mel.go.jp/soshiki/robot/undo/kajita/bipedsitee.html and at http://www.androidworld.com/index.htm. Among all these existing bipeds, the Honda robots seem to be the most advanced at the time of writing of this paper according to the information made available by the owners. However the solution for control designed by Honda does not explain why a given trajectory works nor does it give any insight as to how to select, chain together, and blend various behaviors to effect locomotion through difficult terrain (Pratt (2000)). It is the feeling of the authors that the problem of feedback control of bipedal robots will not be solved properly as long as the dynamics of such systems is not thoroughly understood. In fact, the main motivation for the writing of this paper has been the following observation about walking : there is no analytical study of a stable controller with a complete stability proof available in the related literature. It is our belief that the main reason for this is the lack of a suitable model. We propose a framework that is not only simple enough to allow subsequent stability and control studies but also realistic as some experimental validations prove. In addition, the 
framework provides a unified modeling approach for mathematical, numerical, and control problems, which has been missing. It is for instance significant that the main efforts of the MIT Leg Lab (Pratt (2000)) have been directed toward technological (actuators) improvement and testing of heuristic control algorithms similar to Honda's works.

We would also like to stress that in this survey we made the choice to mainly focus on journal papers. More references can be found by following the links in the above given web pages.

\section{General description of a bipedal walker}

A biped can be represented by an inverted pendulum system that has a constrained motion due to the forward and backward impacts of the swing limb with the ground. Although similar to the structure of vibration dampers in many aspects (Shaw \& Shaw (1989)), which are relatively well studied, structure of bipedal systems have a fundamental difference arising from the unconstrained contact of the limbs with the ground (see Fig. (1)). While the vibration damper remains in contact with the reference frame at all times because of the hinge that is located between the inverted pendulum and the vibrating mass, the limbs of the biped are always free to detach from the walking surface. Detachments occur frequently and lead to various types of motion such as walking, running, jumping, etc. As a matter of fact, one can classify bipedal locomotion systems as complementary slackness systems ((Lötstedt (1984)),(van der Schaft \& Schumacher (1996))). As pointed out below (see Figure (6)), such a modeling framework does not at all preclude the introduction of flexibilities at the contact points. It simply means that the feet can detach from the ground. Also it allows one to include other effects like Coulomb friction in a single framework, which can be quite useful for numerical simulations in order to validate the controllers. Figure (1) depicts other systems that fall into the same category. In the latter part of the article, we will show that such systems can be analyzed by the use of impact-Poincaré maps, and possess common features in terms of motion control. We would like to stress that the focus of this article is motions of such systems that include contact. For example, a biped can rock back and forth while the swing limb

$\mathrm{RR} \mathrm{n}^{\circ} 4290$ 
remains above the walking surface for all times. Such motions will be outside the focus of the discussions presented here.

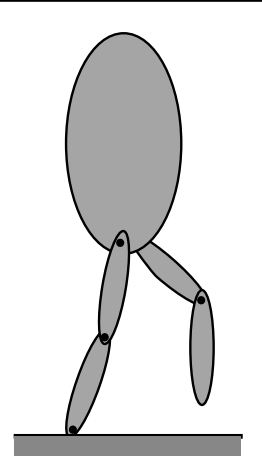

(a)

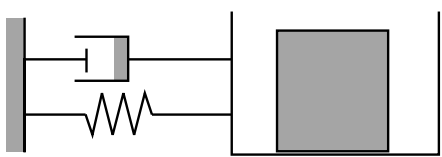

(c)

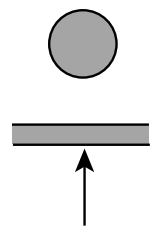

(d)

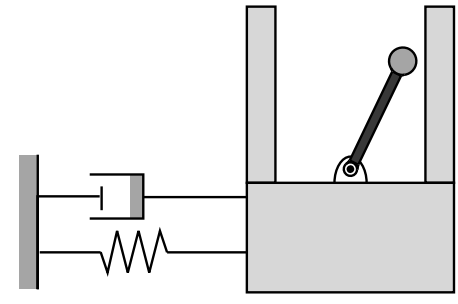

(b)

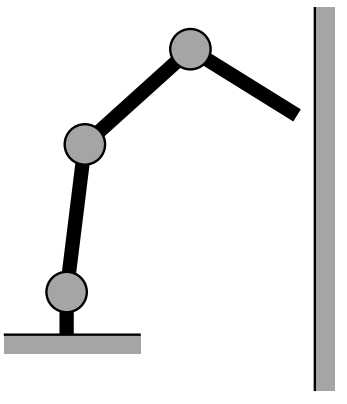

(e)

Figure 1: Bipeds as complementary slackness systems: a) biped, b) pendulum, impact damper, c) mass impact damper, d) juggler, and e) manipulator in contact with a rigid wall

During walking, at least one limb is in contact with the ground at all times. A typical walking cycle may include two phases: the single support phase, when one limb is pivoted to the ground while the other is swinging in the forward direction (open kinematic chain configuration), and the double support phase, when both limbs remain in contact with the ground while the entire system 
is swinging in the forward direction (closed kinematic chain configuration). The impact of the leading limb with the walking surface may lead to two possible velocity outcomes for the tip of the trailing limb : either the trailing limb remains in contact with the ground or it detaches. When both limbs are detached, the biped is in the "flight" phase and the resulting motion is running or some other type of non-walking motion.

Any effort that involves analytical study of the dynamics of gait necessitates a thorough knowledge of the internal structure of the locomotion system. When the system is human or animal, this structure is extremely complicated and little is known about the control strategy that is used by human beings and animals to realize a particular motion and achieve stable gait. If the system represents a man made machine or a numerical model representing such a machine, one has to synthesize control strategies and performance criteria that transform multi-body systems to walking automata.

Devising practical control architectures for bipedal robots, remains to be a challenging problem. The problem is tightly coupled with the control studies in the area of robotic manipulators. Unlike manipulators, however, bipedal machines can have many types of motion. The control objectives should be carefully selected to conform with a specific type of motion. A control strategy that is selected for high speed walking may cause the system to transfer to running, during which an entirely different control strategy should be used.

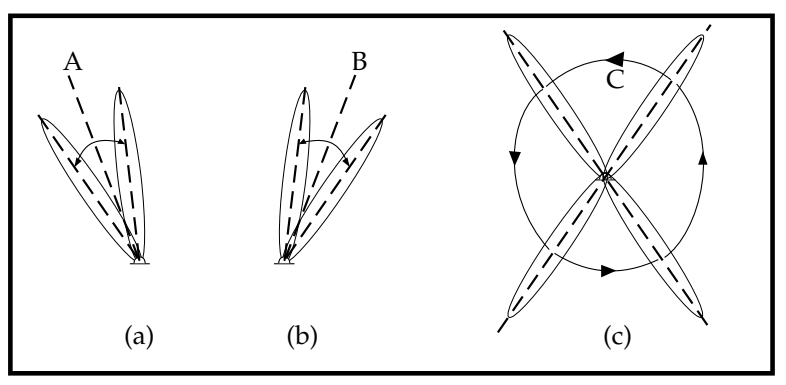

Figure 2: Types of motion: a) Locomotive b) Non-locomotive types of oscillation of a simple pendulum c) Periodic motion

Inverted pendulum models of various complexities have been extensively used in the modeling of gait of humans and bipedal walking machines. Al-

$\mathrm{RR} \mathrm{n}^{\circ} 4290$ 
though, the dynamics of bipedal locomotion is intuitively similar to that of an inverted pendulum, there is a fundamental question that has to be addressed in assessing the validity of the model. The question arises from the inherent instability of inverted pendulums in upright positions. It is well known that one can change the structural stability of inverted pendulum systems by applying torques at various joints. Consider a single degree of freedom model that consists of one mass and a torsional spring at the pivot point. Typical types of motion include oscillations about two static equilibria and a third type where the pendulum undergoes cyclic motions. These three types are depicted in Fig. (2), and labeled as A, B and C respectively. Now, we add two more links to the one link system such that the system looks like the one presented in Fig. (3). When we coordinate the motion of various members by applying appropriate joint moments, we may again observe the three types of motion that we have observed in the single member case (Hurmuzlu \& Moskowitz (1987)). In the presence of the walking surface the system may still be characterized as type A or B, as long as all the parts of the system remain above the ground, Fig. (3.a) and Fig. (3.b). When the system behavior is of type C, however, the swing limb will contact the ground (heel strike) and trigger a chain of events that may lead to stable progression. The importance of this contact event can be better understood if the motion is depicted in the phase space of the state variables.

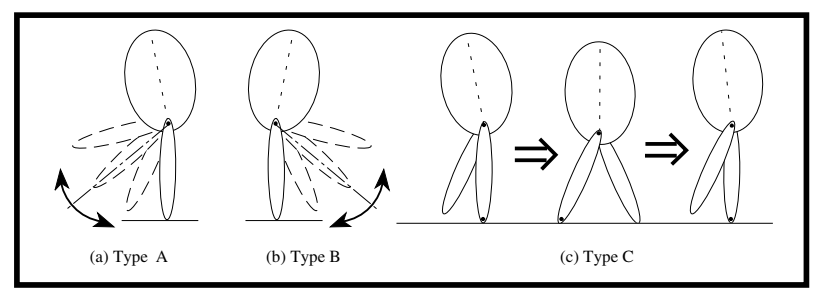

Figure 3: Types of motion of a three element biped

We simplify the present discussion by describing the events that lead to stable progression of a biped for a single degree of freedom system, however this approach can be generalized to higher order models. The phase plane portrait corresponding to the previously described dynamic behavior is depicted in Fig. (4.a). Here, the system may be characterized as a three link biped 
with joint rotations of $\phi_{1}, \phi_{2}$, and $\phi_{3}$. The sample phase plane trajectories corresponding to each type of behavior are labeled accordingly. The vertical dashed lines represent the values of the coordinate depicted in the phase plane for which the contact occurs. For the motions depicted in this figure, the only trajectory that leads to contact is $\mathrm{C}$. The contact event for this simple model produces two simultaneous events:

1. Impact, which is represented by a sudden change in generalized velocities.

2. Switching due to the transfer of pivot to the point of contact.

At this instant, the role of the limbs are exchanged, the old stance limb becomes the new swing limb and the old swing limb becomes the new stance limb. This exchange is classically reflected by sudden changes in the values of generalized positions and velocities. The combined effect of impact and switching on the phase plane portrait is depicted in Fig. (4.b). As shown in the figure, the effect of the contact event will be a sudden transfer in the phase from point 1 to point 2, which is generally located on a different dynamic trajectory than the original one. If the destination of this transfer is on the original trajectory, then the resulting motion becomes periodic (i.e. a limit cycle as shown in Fig. (4.c)).

Another graphical construction of the evolution of the state of the system is depicted in Fig. (4.d). This construction is obtained by first choosing a Poincaré section (in case of bipedal locomotion a well defined instant during the gait cycle). For example, let us assume that we choose the heel strike as the Poincare section. Then, we can obtain a sequence of points constructed by pairing the value of a state variable at the heel strike of the $i^{\text {th }}$ cycle with the value of the same variable at the heel strike of the $i+1^{\text {th }}$ cycle. A typical plot of this sequence is depicted in Fig. (4.d)), which is called a Poincaré map. In the figure, each pair is labeled as $p_{i}$. The iteration point $p_{e}$ that corresponds to the closed orbit of Fig. (4.c)) is on the $45^{\circ}$ degree line because when the motion is periodic $\phi_{1}$ at heel strike is identical for all successive locomotion steps. In the case of stable locomotion, the state of the system approaches the steady state as shown in the figure. We may observe that points on the map accumulate at $p_{e}$ as the biped takes successive locomotion steps toward the equilibrium. The same construction can also be performed for the generalized velocity $\dot{\phi}_{1}$ or for 
any other kinematic quantity. This type of periodicity has unique advantage when the inverted pendulum system represents a biped. Actually, this is the only mode of behavior that this biped can achieve progression. The most striking aspect of this particular mode of behavior is that the biped achieves periodicity by utilizing only a portion of a dynamic trajectory. The impact and switching modes provide the connection between the cyclic motions of the kinematic chain and the walking action.

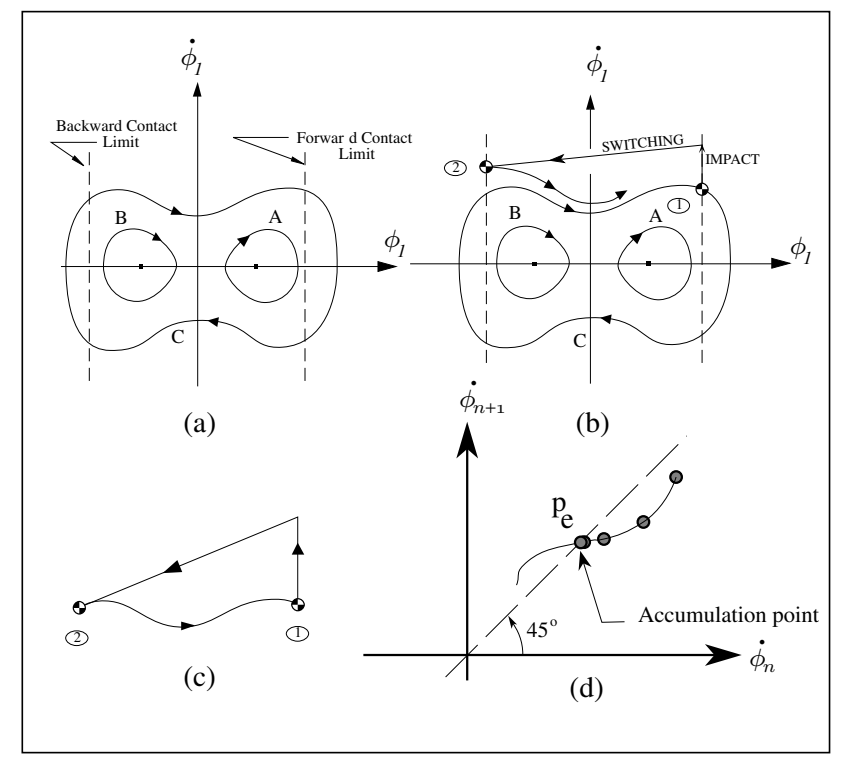

Figure 4: Impact and switching on phase plane portrait

We can clearly observe from the preceding discussion that the motion of a biped involves continuous phases separated by abrupt changes resulting from impact of the feet with the walking surface. During the continuous phase, we may have none, one, or two feet in simultaneous contact with the ground. In the case of one or more feet contacts, the biped is a dynamical system that is subject to unilateral constraints. Systems with unilateral constraints are systems whose configuration space is $S$, and they are not not allowed to penetrate into a closed domain $W \subset S$. Under some regularity conditions the admissible domain $\Phi=S \backslash W$ can be described by a set of inequalities 
$F(q) \geq 0$ (i.e. the intersection of the domains $\left.F_{i}(q) \geq 0, i=1,2, \ldots, m\right)^{1}$. For example, in bipedal locomotion systems, the closed domain $W$ is the ground that the biped walks on. The contacts between the feet and the walking surface can be characterized as unilateral constraints. The inequalities $F_{i}(q) \geq 0$ describe the nonpenetration conditions. Consequently, when a foot impacts the ground surface, we face the impact problem of a multi-link chain with unilateral constraints. In fact, the overall motion of the biped may include a very complex sequence of continuous and discontinuous phases. This poses a very challenging control problem, with an added complication of continuously changing motion constraints and large velocity perturbations resulting from ground impacts.

\section{Mathematical Description of a biped as a sys- tem subject to unilateral constraints}

\subsection{Dynamics}

Bipedal locomotion systems are "unilaterally constrained" dynamical systems (ten Dam (1997)). A way to model such systems is to introduce a set of unilateral constraints in the following form:

$$
F(q) \geq 0, q \in \mathbb{R}^{p}, F: \mathbb{R}^{p} \longrightarrow \mathbb{R}^{m}
$$

where $q$ represents the complete vector of generalized coordinates, that is, $p$ denotes the number of degrees of freedom of the system without constraints, i.e. when $F(q)>0$. The constraints mean that the bodies that constitute the system cannot interpenetrate. For a rigid biped robot, $q$ could be chosen to be the complete vector of joint coordinates plus the euclidian space coordinates of a point attached to the robot (typically, one extremity of the kinematic chain), whereas, $F(q)$ could be reduced for practical reasons to the relative feet to ground distances. The dynamics of a $p$-degree-of-freedom mechanical system subject to $m$ unilateral constraints may be written as the following

\footnotetext{
${ }^{1} \mathrm{~A}$ sufficient regularity conditions are: the boundary of $\Phi$ is of codimension 1 , or $m \geq 2$ and $\Phi$ is convex.
}

$\mathrm{RR} \mathrm{n}^{\circ} 4290$ 
system $(\mathcal{S})$ of equations :

$$
\begin{aligned}
& M(q) \ddot{q}+N(q, \dot{q})=T u+\nabla F(q) \lambda_{n}+P_{t}(q, \dot{q}) \\
& \lambda_{n}^{T} F(q)=0, \quad \lambda_{n} \geq 0, \quad F(q) \geq 0 \\
& \text { Restitution law }+ \text { shock dynamics } \\
& \text { Dry Friction Amontons-Coulomb's model }
\end{aligned}
$$

where $M(q)$ is the inertia matrix, $N(q, \dot{q})$ includes Coriolis, centrifugal, gravitational, and other terms, $T u$ is an external input. The rest of the terms and variables are defined next.

For the bipeds the Lagrange dynamics can be rewritten in a specific fashion that corresponds to control objectives and allows the designer to get a better understanding of their dynamical features (Wieber (2000); Grizzle, Abba \& Plestan (2001)). In other words, the choice of the generalized coordinates $q$ is crucial for control purpose, and certainly much less obvious than it is for serial manipulators. For example in Fig. 5, $q$ can be split in two subsets $q_{1}$ and $q_{2}$. The vector $q_{1}=(x, y, \theta)$ describes the global position of the robot in space whereas the vector $q_{2}=\left(\alpha_{1}, \ldots, \alpha_{6}\right)$ are the joint coordinates. The vector $q_{1}$ could be attached to any point of the biped. Nevertheless it is known from biomedical studies of human gait that one of the primary objectives during locomotion is the stabilization of the head where the exteroceptive sensors (inner ear, sight) are located. Setting $q=\left(q_{1}, q_{2}\right)^{T},(1)$ splits in an upper part and a lower part corresponding to head motion and joint dynamics respectively :

$$
\begin{aligned}
\left(\begin{array}{c}
M_{1}(q) \\
M_{2}(q)
\end{array}\right) \ddot{q}+\left(\begin{array}{c}
N_{1}(q, \dot{q}) \\
N_{2}(q, \dot{q})
\end{array}\right) & =\left(\begin{array}{c}
0 \\
T_{2}
\end{array}\right) u+\nabla F(q) \lambda_{n}+P_{t}(q, \dot{q}) \\
F(q) & =\left(y_{A_{1}}, y_{B_{1}}, y_{A_{2}}, y_{B_{2}}\right)^{T} \in \mathbb{R}^{4}
\end{aligned}
$$

In the sequel (section 3.2.2) we will review the available modeling tools whcih will allow the designer to complete (5) with (3) and (4). Here $T_{1}=0$ since the biped has only joint actuators. As a matter of fact head motion can only be achieved thanks to a coordinated action of joint actuations and contact forces. This fact is probably more apparent for the flight phases (running) during which there are no contact forces and the trajectory of the center of mass (attaching this time $q_{1}$ to the center of mass) yields only to gravity. 


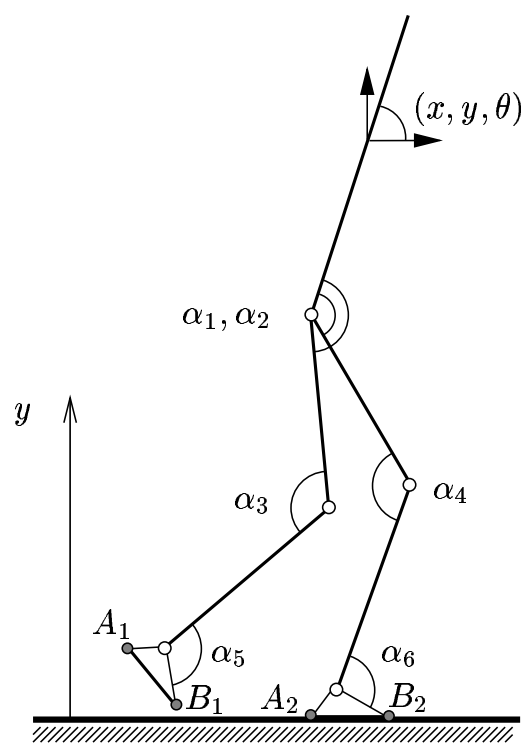

Figure 5: A 9 DoF Planar Biped

All complementarity-slackness systems depicted in Fig. (1) can be represented in the form given by (1)-(4). For example, the first order dynamics of a juggler can be written as :

$$
\left\{\begin{array}{l}
\dot{z}_{1}=f_{1}\left(z_{1}, t, \lambda\right) \\
\dot{z}_{2}=f_{2}\left(z_{2}, t, u, \lambda\right) \\
\lambda^{T} h\left(z_{1}, z_{2}\right)=0, \lambda \geq 0, \quad h\left(z_{1}, z_{2}\right) \geq 0 \\
\text { Collision mapping }
\end{array}\right.
$$

The $z_{1}$-dynamics represents the dynamics of the "object" (which may be a real object like a puck, or the centre of gravity dynamics of a flying system like when the biped is airborne (Brogliato (1999)), while the $z_{2}$-dynamics is that of the "robot". The analogy between (7) and (5) (2) (3) (4) can be made where $z_{1}$ is the center of gravity coordinates.

However biped dynamics possesses some specific features that make the control study differ significantly from the control of other complementarityslackness systems (see Fig. (1)). Bipeds share the following features :

$\mathrm{RR} \mathrm{n}^{\circ} 4290$ 
1. With systems (b),(d) and (e) : the center of mass is not controlled when $F(q)>0$ (as the object of the juggler in (7)),

2. With system (e) : their dynamics is that of a manipulator when one foot sticks to the ground,

3. With system (b) : they may be underactuated (no ankle actuator) and act as an inverted pendulum when one foot sticks to the ground.

The fact that bipeds merge all these characteristics makes their control analysis complex. In this survey we only consider systems with lumped flexibilities (systems with distributed flexible effects are not considered here). Equation (1) represents the smooth dynamics when either the system evolves in freemotion or in a phase of permanent contact, i.e.

$$
\begin{cases}F_{i}(q) \equiv 0 & \text { for some } i \in \mathcal{I}(q) \subseteq\{1, \ldots, m\} \\ F_{j}(q)>0 & \text { for } j \notin \mathcal{I}(q)\end{cases}
$$

The set of equations in (2) represents the complementarity conditions between the normal force $\lambda_{n} \in \mathbb{R}^{m}$ and the position. Then, in equation (1) $\left(\nabla F_{i}=\frac{\partial F_{i}^{T}}{\partial q} \in \mathbb{R}^{p}\right.$ is the gradient vector $)$ :

$$
\nabla F \lambda_{n}=\Sigma_{i=1}^{i=m} \nabla F_{i} \lambda_{n, i}
$$

Notice that for $i \in \mathcal{I}(q)$, one can express (2) as

$$
\lambda_{n, i} \dot{F}_{i}(q)=0, \quad \lambda_{n, i} \geq 0, \quad \dot{F}_{i}(q) \geq 0
$$

or, if $\dot{F}_{i}(q) \equiv 0$ for $i \in \mathcal{I}(q)$, as:

$$
\lambda_{n, i} \ddot{F}_{i}(q)=0, \quad \lambda_{n, i} \geq 0, \ddot{F}_{i}(q) \geq 0
$$

One may see Signorini's conditions (Jean (1993)) for other formulation of the complementarity relations. We should notice that (2), (9) and (10) are not equivalent. Indeed one may have $F_{i}\left(q\left(\tau_{0}\right)\right)=0$ and $\dot{F}_{i}\left(q\left(\tau_{0}\right)\right)>0$ or $\dot{F}_{i}\left(q\left(\tau_{0}\right)\right)=$ 0 and $\ddot{F}_{i}\left(q\left(\tau_{0}\right)\right)>0$. In the first case, following (2), $\lambda_{n, i} \geq 0$ while from (10), $\lambda_{n, i}=0$, and similarly for the second case. More precisely let us investigate 
the case where an active constraint $i$ becomes passive at $t=\tau_{0}$, see (Lötstedt $(1982))$. On $\left(0, \tau_{0}\right]$, one has :

$$
\begin{aligned}
F_{i} & =0, \\
\dot{F}_{i} & =\nabla F_{i}^{T} \dot{q}=0, \\
\ddot{F}_{i} & =\nabla F_{i}^{T} \ddot{q}+f(q, \dot{q})=0 .
\end{aligned}
$$

The fact that the active constraint $i$ becomes passive at $\tau_{0}$ means that there exists $\epsilon_{0}>0$ such that, on $\left(\tau_{0}, \tau_{0}+\epsilon_{0}\right), F_{i}>0$. Now :

$$
F_{i}(t)=\nabla F_{i}^{T}\left(\tau_{0}\right) \dot{q}\left(\tau_{0}\right)\left(t-\tau_{0}\right)+O\left(\left(t-\tau_{0}\right)^{2}\right)>0
$$

Thus, there exists $\epsilon_{1}>0, \epsilon_{1}<\epsilon_{0}$, such that on $\left(\tau_{0}, \tau_{0}+\epsilon_{1}\right), \dot{F}_{i}>0$. Similarly there exists $\epsilon_{2}>0, \epsilon_{2}<\epsilon_{1}$, for which on $\left(\tau_{0}, \tau_{0}+\epsilon_{2}\right), \ddot{F}_{i}>0$. This shows that $(2)$ is valid on $(0,+\infty),(9)$ on $\left(0, \tau_{0}+\epsilon_{1}\right)$ and $(10)$ only on $\left(0, \tau_{0}+\epsilon_{2}\right)$. Let us note here that this process of successive derivation can be reiterated ad libitum : Van der Schaft et al. (van der Schaft \& Schumacher (1996)) speak of DCP ("Dynamical Complementarity Problem"). Kunze and Neumann (Kunze $\&$ Neumann (1997)) use similar arguments to stabilize the index set in $(\mathcal{S})$ by including higher order derivatives in their definition.

The advantage of (10) is that using (1), it allows to obtain a LCP ("Linear Complementarity Problem") whose unknown is the interaction force. The calculation of a unique $\lambda_{n}$ at $\tau_{0}^{-}$is in turn a sufficient condition for the integration (for instance numerically) of $(\mathcal{S})$ on $\left(\tau_{0}, \tau_{0}+\epsilon\right)$ for a $\epsilon>0$ small enough, see (Moreau (1985)). However the integration can be treated in an implicit form without the explicit calculation of the contact forces, as proposed by the "sweeping process", see (Moreau (1986)) and section 3.2 for an introduction: $\dot{q}\left(\tau_{0}^{+}\right)$is computed directly from $\dot{q}\left(\tau_{0}^{-}\right)$and the generalized forces $T u-N(q, \dot{q})$ adding a projection procedure that guarantees non-violation of the constraints at $\tau_{0}^{+}$. Finally the models in (3) and (4) are needed to complete the dynamics. In particular it is necessary to relate the post-impact velocities to the pre-impact data to be able to compute solutions that are compatible with the constraints (integrate the system and render the domain $\Phi \triangleq\{q \mid F(q) \geq 0\}$ invariant). The classical dry friction model in (4) provides the form of $P_{t}(q, \dot{q})$ in (1), see section 3.3.

$\mathrm{RR} \mathrm{n}^{\circ} 4290$ 
Now, if we also consider the complementarity relationship, compliant interfaces can be easily cast in the form given by (2). For example, let us consider the system shown in Fig. (6). For this simple case the complementarity will be given by:

$$
\begin{gathered}
F d=0, \quad F \geq 0, d \geq 0 \\
d=x_{m}-x_{e}
\end{gathered}
$$

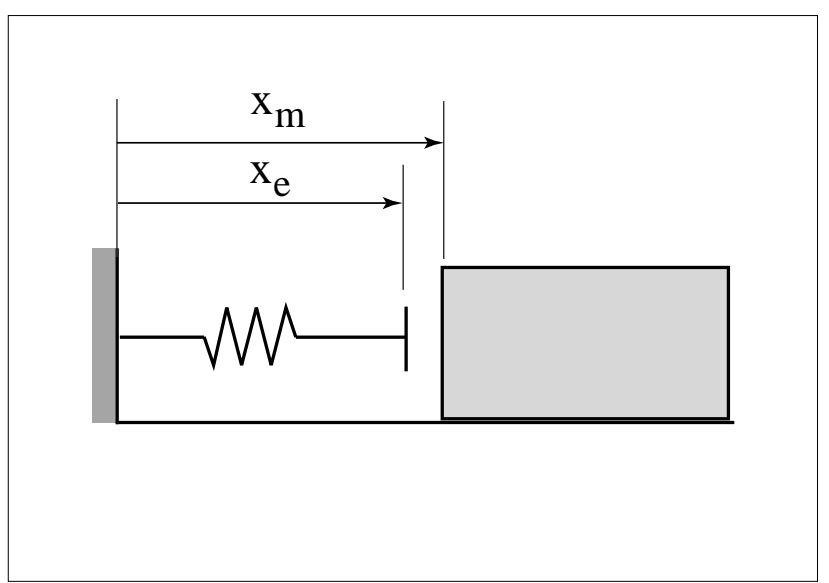

Figure 6: Flexible contact

We should note that the example is given to demonstrate that complementarity conditions can accommodate flexible contacts. In the ensuing part of the paper, however, we choose to deal only with rigid contact. The main reason for such a choice is that the rigid-body approach provides a model that is suitable for both control design and analysis. Actually most of the studies on bipeds control rely on a rigid-body approach with impulsive forces at impacts with the ground. It is therefore quite fundamental, before going to the control design, to have a clear understanding of the underlying dynamics in order to make accurate numerical simulations (Brogliato, ten Dam, Paoli, Génot \& Abadie (2001); Abadie (2000)). Finally the rigid-body complementarity approach has been validated experimentally in several instances (Abadie (2000); Pfeiffer \& Glocker (1996)). It is also important to notice that lumped flexibilities can 
be introduced in (1) (for instance stiffnesses at the knees), and would help in better modeling the vibrations created by the impacts. Clearly however the control problem complicates in this case. Consequently the model in (1)-(4) is very general and in particular encompasses all the models which have been proposed in the literature on bipeds control.

It is apparent that the set of equations in (1)-(10) defines a complex hybrid dynamical system, in the sense that it mixes both continuous and discreteevent phenomena. The states of the discrete-event system (DES) are defined by the $2^{m}$ modes of the complementarity conditions in (2). Integration of such systems is more difficult than that of ODEs or DAEs. Indeed assume that the initial continuous state $\left(q_{0}, \dot{q}_{0}\right)$ belongs to a certain mode (that we may denote mode $\mathcal{I}\left(q\left(t_{0}\right)\right)$ from (8)) and that the corresponding DAE is well-posed. (It seems that the least requirement for well-posedness of $(\mathcal{S})$ is that to each mode $\mathcal{I}(q)$ there corresponds a well-posed systems of DAE, see (van der Schaft \& Schumacher (1996))). Then it may happen that $(q(t), \dot{q}(t))$ reaches a boundary between two (or more) possible modes : one active constraint $\left(F_{i}(q)=0\right)$ may become inactive $\left(F_{i}(q)>0\right)$ while one or several others which were inactive, become active. Is such a transition smooth (i.e. the continuous state attains the boundary in such a way that it "naturally" (or "tangentially") enters in another mode? Or is it needed to abruptly modify the continuous vector field direction (state jump)? Is the continuation of the continuous trajectory unique and in a unique mode? There may be cases in which the trajectory is unique but belongs to several modes, i.e. evolves in a codimension $\geq 2$ subspace of the configuration space corresponding to an indeterminate case (van der Schaft \& Schumacher (1996)) ; there may also be cases in which several trajectories $(q(t), \dot{q}(t))$ emerge from one state, related to different DES states, even if the system is autonomous - (van der Schaft \& Schumacher (1996)), example 4.2 or if the contact forces $\lambda_{n}$ are uniquely determined (Bressan (1959)).

It is also important to point out that such systems fundamentally differ in nature from those studied in (Bainov \& Simeonov (1989)), which consist of mainly ordinary differential equations with impulsive disturbances.

In the sequel we shall try to survey the principal contributions that concern

1. Particular models of multiple impacts in (3) with or without friction,

RR $n^{\circ} 4290$ 
2. The study of existence of solutions problems for LCPs related to (2) with and without friction,

3. The study of existence and uniqueness of solution to the whole hybrid dynamical system $(\mathcal{S})$ in $(1)-(4)$.

From a mechanical engineer point of view, two questions have to be answered to, when one wants to integrate the system (1)-(4) (Moreau (1988)):

Q1 Assume that $\mathcal{I}(q)$ is non empty at $\tau_{0}$, and that the velocity $\dot{q}\left(\tau_{0}^{-}\right)$points inwards $\Phi$ or tangentially to $\partial \Phi^{2}$ : Determine which contacts $i \in \mathcal{I}(q)$, will persist at $\tau_{o}^{+}$. In other words determine the subsequent mode (or DES state).

Q2 At $t_{k}$, one has $\dot{q}\left(t_{k}^{-}\right)^{T} \nabla F_{i}\left(q\left(t_{k}\right)\right)<0$ for some $i \in\{1, \ldots, m\}$ and $F_{i}\left(q\left(t_{k}\right)\right)=$ 0 . Thus a shock occurs at $t_{k}$. Determine the right velocity $\dot{q}\left(t_{k}^{+}\right)$. In other words determine a re-initialization (van der Schaft \& Schumacher (1996)) of the (continuous) state as soon as one (or several) of the complementarity conditions in (2) is going to be violated.

In the sequel $t_{k}, k=0,1,2 \ldots$ will generically denote the successive impact instants.

The answer to those questions is far from being trivial and has been the object of many researches. The first one is related to solving the LCP associated to the system, i.e. being able to calculate at each time of a collision-free phase the interaction forces $P_{q} \triangleq \nabla F(q) \lambda_{n}+P_{t}(q, \dot{q})$, hence the acceleration $\ddot{q}$ and the subsequent motion. It has been raised initially by Delassus (Delassus (1917)) (see also (Pfeiffer \& Glocker (1996)) for a very nice and simpler example). The second question is that of defining proper restitution rules, or collision laws. This goes back to the $17^{\text {th }}$ century and the celebrated Newton's conjecture, see (Brogliato (1999); Kozlov \& Treshchev (1991)) for more details. In particular if several hypersurfaces $\Sigma_{i}=\left\{q \mid F_{i}(q)=0\right\}$ are attained simultaneously (or if the system rebounds indefinitely on several $\Sigma_{i}$ and with a finite accumulation

\footnotetext{
${ }^{2}$ In the case of non-differentiable $\partial \Phi$, corresponding to $m \geq 2$, one has to extend the notions of tangent and normal spaces to tangent and normal cones, see (Moreau (1988)).
} 
of impact times $t_{k}$ ), a multiple impact occurs. ${ }^{3}$ Such an event occurs typically during walking at the end of a single support phase when the swinging leg foot hits the ground. For example, for the planar biped depicted in Figure 5, a 3 -impact takes place at foot strike $t_{k}$, that is

$$
\begin{aligned}
& y_{A_{2}}(t)=y_{B_{2}}(t)=0, y_{A_{1}}(t)>0, y_{B_{1}}(t)>0, \text { for } t<t_{k} \\
& y_{A_{2}}\left(t_{k}\right)=y_{B_{2}}\left(t_{k}\right)=0, y_{A_{1}}\left(t_{k}\right)=0, \dot{y}_{A_{1}}\left(t_{k}^{-}\right)<0 .
\end{aligned}
$$

Defining restitution laws for multiple impacts such that the system (1)(4) is well-posed is not trivial (even the case of a simple impact between two bodies is not evident when friction is non-negligible, see (Brach (1991); Stronge (1990))), see (Ivanov (1995)).

In summary, the hybrid dynamical system in (1)-(4) (or (1)-(3),(10)) requires, in order to be integrated, that both questions Q1 and Q2 above be answered, and that the used models yield well-posedness. This means that one should be able to calculate in a unique manner, at every time $t$, the state evolution. This may be done in particular through the study of LCPs, i.e. calculation of contact forces.

The approach one may have in order to solve this problem depends on one's objectives. It happens that researchers from computer science (Baraff (1993); Baraff (1994); Lötstedt (1984)), applied mathematics (Lötstedt (1982); Trinkle, Pang, Sudarsky \& Lo (1995); Pang \& Trinkle (1996); Paoli \& Schatzman (1993); Paoli (1993); Monteiro-Marques (1993); Stewart \& Trinkle (1996); Kunze \& Neumann (1997)), robotics (Trinkle, Pang, Sudarsky \& Lo (1995); Pang \& Trinkle (1996); Dupont (1992)), mechanics (Moreau (1988); Stronge (2000); Han, Gilmore \& Ogot (1993b); Haug, Wu \& Yang (1986); Pfeiffer \& Glocker (1996); Han \& Gilmore (1993)), control theory (Brogliato, Niculescu \& Orhant (1997); Brogliato \& Zavala-Rio (2000); Zavala-Rio \& Brogliato (1999); Brogliato, Niculescu \& Monteiro-Marques (2000); Tornambe (1999); van der

\footnotetext{
${ }^{3}$ Such phenomena should not be confused with so-called micro-collisions. Micro-collisions correspond to a series of sequential impacts between two bodies $(m=1)$. They result from the flexibilities and the subsequent vibrations induced by the shock in the bodies. At a macroscopic level the duration of the whole contact phase may be very short and considered as being instantaneous (Marghitu \& Hurmuzlu (1996); Stoianovici \& Hurmuzlu (1996); Hurmuzlu (1998)). Micro-collisions are sometimes called multiple-collisions in the literature, hence possible confusion.
}

RR $n^{\circ} 4290$ 
Schaft \& Schumacher (1996); ten Dam, Dwarshuis \& Willems (1997)) have recently made significant advances concerning dynamical systems with unilateral constraints. In the following, we provide a brief survey of those recent works.

As we pointed out earlier, the integration, modeling and control of systems like $(\mathcal{S})$ has recently interested researchers of different horizons. Despite the fact that their goals may differ as well, the fundamental problem is always to be able to integrate the trajectory, starting from admissible initial data $q(0), \dot{q}(0)$. Clearly existence and uniqueness of solution of $(\mathcal{S})$ is a basic and quite useful property to that aim. The following survey part does not aim at making a classification among those works. It just aims at providing an outline of this topic. Since in this survey we focus on bipedal robots, we shall essentially describe those works that handle the case of multiple simultaneously active constraints. In other words restitution laws and coefficients for the basic case of two colliding bodies will not be discussed in the following : the interested reader may consult (Brach (1991); Brogliato (1999); Stronge (2000)) and references therein.

Before going any further into the details of the dynamics of systems subject to unilateral constraints, let us first stress that from our point of view only too few attention was payed in the literature to the existence of those constraints. In fact most of the works on the control of biped robots model the robot in the single support phase as a manipulator whose base corresponds to the supporting foot and add some closed loop constraints for the double support phase. Even if this approach is very convenient to derive trajectory tracking laws via the computed torque technique, it doesn't account for possible slippage nor detachment at ground contacts nor say anything about the influence of impacts on stability :

- The contacts are assumed to be bilateral during each support phase, i.e. $F(q)=0$ in the normal direction and no slip in the tangential contact directions,

- The only support phase transitions are from sticking single support towards sticking double support and vice versa.

In other words, fundamental events like detachments or foot slippage are not considered. Notice however that the human walking pattern and the under- 
lying control strategy differs notably depending on the ground characteristics (ice arena, basketball playground, trampoline, etc.). As shown in appendix $\mathrm{A}$ and appendix $\mathrm{B}$, the control torques $u$ have to obey certain conditions to ensure sticking at all times. These conditions can be written as (Génot (1998); Génot, Brogliato \& Hurmuzlu (1998)):

$$
A(q, \mu) u+B(q, \dot{q}, \mu)>0
$$

during smooth motion, and as:

$$
\bar{A}\left(q\left(t_{k}\right), \mu\right) \dot{q}\left(t_{k}^{-}\right)>0
$$

at impact times (and for some choice of the double impact model), where $\mu$ is Amontons-Coulomb's friction coefficient at contact. Both inequalities in (11) and (12) are necessary and sufficient conditions to be satisfied by the control input $u$ so that during the whole motion (smooth and nonsmooth) sticking is maintained and detachment is monitored. To the best of our knowledge, none of the control laws proposed in the literature until now was shown to be "stable" with respect to these fundamental conditions, mainly due to the fact that the underlying dynamics of the robot doesn't capture the unilateral feature of the feet-ground contacts.

\subsection{Frictionless contacts}

The succeeding subsections are devoted to the study of the LCP in (10) (i.e. the calculation of the contact forces) and multiple impacts respectively.

\subsubsection{Continuous motion}

In the frictionless case $(\mathcal{S})$ reduces to $(1),(2)$ and $(3)$ with $P_{t}(q, \dot{q})=0$. Notice that the complementarity relation (2) is natural since it expresses that there is no force of attraction or adhesion between two contacting bodies. In particular Pérès (Pérès (1953)) has shown that in the case $m=1$ the dynamical equation (1) together with the unilaterality of displacement, that is $\dot{F}(q) \geq 0$ or $\ddot{F}(q) \geq$ 0 , implies that the bodies cannot detach as long as $\lambda_{n}>0$. To the best of our knowledge this is the only case where the complementarity relation (2) was effectively proven to be necessary. Moreau (Moreau (1963); Moreau (1966))

$\operatorname{RR} \mathrm{n}^{\circ} 4290$ 
has been the first to show that in the multiple constraints case $m \geq 1$, using that $\ddot{F}=\nabla F^{T} \ddot{q}+f(q, \dot{q})$, the complementarity relation (10) combined with (1), setting $h(q, \dot{q})=T u-N(q, \dot{q})$, yields a Linear Complementarity Problem (LCP) of the form

$$
\ddot{F}=A \lambda_{n}+b \geq 0, \quad \lambda_{n} \geq 0 \text { and } \lambda_{n} \ddot{F}=0
$$

where

$$
A=\nabla F^{T} M^{-1} \nabla F
$$

and

$$
b=\nabla F^{T} M^{-1} h(q, \dot{q})+f(q, \dot{q})
$$

The interested reader may have a look at (Cottle, Pang \& Stone (1992)) for an introduction and general results on LCPs. If the active constraints $(i \in \mathcal{I}(q))$ are independent, then $A$ is positive symmetric definite (PSD) and it is known that the LCP in (13) possesses a unique solution $\lambda_{n}$ (Cottle \& Dantzig (1968)). If some constraints are dependent, then $A$ is only semi-PSD and uniqueness only holds for the acceleration $\ddot{F}$. Moreover $\nabla F \lambda_{n}$ is also unique, see (Moreau (1966); Lötstedt (1982)) Lemma 5.2, and thus from the dynamics (1) $\ddot{q}$ is unique too. As a classical example one may think of a chair with four legs on a rigid ground : even if the interaction forces cannot be uniquely determined the acceleration of the mass center is unique (upwards).

Using Kuhn-Tucker's theory (Kuhn \& Tucker (1951)) it is possible to show that any solution $\lambda_{n}$ to the LCP in (13) is also a solution to the quadratic problem

$$
\min _{\lambda_{n} \geq 0} \frac{1}{2} \lambda_{n}^{T} A \lambda_{n}+\lambda_{n}^{T} b
$$

From (Dorn (1960)) this is in turn equivalent to solving the dual problem

$$
\min _{\lambda_{n}} \frac{1}{2} \lambda_{n}^{T} A \lambda_{n}, \quad A \lambda_{n}+b \geq 0
$$

Substituting $\nabla F \lambda_{n}$ by $M \ddot{q}+N(q, \dot{q})-T u$, see (1), in (15) one straightforwardly obtains

$$
\min _{\ddot{q}} \frac{1}{2}\left(\ddot{q}-M^{-1} h(q, \dot{q})\right)^{T} M\left(\ddot{q}-M^{-1} h(q, \dot{q})\right), \quad \nabla F \ddot{q}+f(q, \dot{q}) \geq 0
$$


which is known as Gauss' principle ("least deviation") (Moreau (1966); Lötstedt (1982)).

From the programming point of view it must be noted that except in the last decade the Mathematics community paid more attention to developing general algorithms for QPs than for the more particular class of the LCPs. Hence the interest of the above cited equivalences.

Finally let us note that recent works propose to solve LCPs, or more generally NCPs ("Nonlinear Complementarity Problems"), through unconstrained optimization (Fischer (1992); Kanzow (1996)), by introducing so-called NCP functions $\phi: \mathbb{R}^{2} \mapsto \mathbb{R}$ satisfying the complementarity condition

$$
\phi(a, b)=0 \Leftrightarrow a \geq 0, \quad b \geq 0, \quad a b=0
$$

For instance admissible functions are

$$
\begin{aligned}
& \phi(a, b)=\frac{1}{2} \min ^{2}(a, b) \\
& \phi(a, b)=\frac{1}{2}\left(\sqrt{a^{2}+b^{2}}-a-b\right)^{2} .
\end{aligned}
$$

In our case $a=\ddot{F}$ and $b=\lambda_{n}$ are vectors of dimension $z=\operatorname{card}(\mathcal{I}(q))$, that is the number of elements in the set $\mathcal{I}(q)$. Let us define the function

$$
\Psi: \mathbb{R}^{z} \mapsto \mathbb{R}, \Psi\left(\lambda_{n}\right)=\sum_{i \in \mathcal{I}(q)} \phi\left(\lambda_{n, i}, \ddot{F}_{i}\left(\lambda_{n}\right)\right)
$$

The proposed algorithms base on the following lemma

Lemma 1 Assume that the complementarity problem is solvable and that $\phi$ is a NCP function with $\phi(a, b) \geq 0, \forall(a, b) \in \mathbb{R}^{2}$. Then

$\lambda_{n}^{*}$ is a global minimum of $\Psi \Leftrightarrow \lambda_{n}^{*}$ solves the complementarity problem.

More information on LCPs, NCPs, and their domains of application can be found in (Ferris \& Pang (1997)).

Now to summarize, it is clear that if one is able to obtain at time $\tau_{0}^{-}$ a value for $\lambda_{n}$, then introducing this value into (2) allows one to determine which contact persist at $\tau_{0}^{+}$and which are going to break (become inactive) on $\left(\tau_{0}, \tau_{0}+\epsilon\right), \epsilon>0, \epsilon$ small enough.

RR $n^{\circ} 4290$ 
Remark 1 As we pointed out in the introduction, the answer to Q1 does not necessarily require the explicit calculation of the contact forces $\lambda_{n}$. Let us consider the simple example $(p=1)$ of a ball resting on the ground $(m=1)$ at $\tau_{0}^{-}$. Suppose that an external force $f$ is applied to the ball at $\tau_{0}$. Then the acceleration $\ddot{q}$ is given by

$$
\ddot{q}=\max (0, f)
$$

Let us discretize the unconstrained motion $(q>0)$ as

$$
\left\{\begin{array}{l}
q_{i+1}=q_{i}+h \dot{q}_{i} \\
\dot{q}_{i+1}=\dot{q}_{i}+h f
\end{array}\right.
$$

Using (21), (20) can be rewritten as

$$
\dot{q}_{i+1}=\operatorname{prox}\left[\mathbb{R}^{+}, \dot{q}_{i}+h f\right]
$$

where prox denotes the proximal point to $\dot{q}_{i}+h F$ in $\mathbb{R}^{+}$.

This implicit scheme can be generalized to more general systems $(p \geq$ $1, m \geq 1$ ), as proposed by Moreau (Moreau (1986)). Moreover it can be generalized to provide one solution to the multiple impact problem as will be shown in the next subsection.

\subsubsection{Multiple impacts}

The foregoing paragraph was devoted to partially answer to Q1 above. We now focus on Q2. In general walking robots use several support points during locomotion (biped robots (Hurmuzlu \& Moskowitz (1986); Hurmuzlu \& Moskowitz (1987); Hurmuzlu (1993)), quadruped robots (Chevallereau, Formal'sky \& Perrin (1997); Perrin, Chevallereau \& Formal'sky (1997))). In other words $m \geq 2$. This means that the boundary $\partial \Phi$ of the admissible domain $\Phi$ is not regular. Its singularities correspond to surfaces with codimension $\geq 2$. Thus the eventuality that the state collides in a neighbourhood of a singularity cannot be excluded in mechanical systems as $(\mathcal{S})$. In the framework of bipedal locomotion such events intrinsically belong to the dynamics of walking. For instance consider the problem of a kinematic chain of rigid bodies which collides with the ground at one point $A_{j}, j \notin \mathcal{I}(q)$ while still having several support points $A_{i}, i \in \mathcal{I}(q)$. More formally there exists an interval $T=\left[t_{k}-\epsilon, t_{k}\right)$ such 
that $\mathcal{I}(q)$ remains constant on $T$ while $\exists j \notin \mathcal{I}(q) \mid \dot{q}\left(t_{k}^{-}\right)^{T} \nabla F_{j}\left(q\left(t_{k}\right)\right)<0$ and $F_{j}\left(q\left(t_{k}\right)\right)=0$. The main and not trivial problem is to find out in which way the impact at $A_{j}$ will influence the points $A_{i}$ : may the points initially in contact detach from the ground and what happens at $A_{j}$ ? Since again we concentrate on bipedal robots we shall take into account that the collision events of such robots mainly correspond to collisions of the swinging foot with the ground. It is not our aim to discuss here the classical assumption that these shocks are inelastic but rather to present the different approaches proposed in the literature that allow one to solve this problem from a general point of view. In other words let us view for simplicity the bipedal robot as a point $R(t)$ in a two-dimensional generalized configuration space. The classical standard walking assumption can be seen as a "bilateral sliding motion" of the generalized point $R(t)$ confined to the two constraint boundaries, see Figure 7(a). Single support phases correspond to $R(t) \in[A, B) \cup(B, C]$, the double support phase to $R(t)=B$. However one cannot a priori exclude

- rebounding multiple shocks at $B$, see Figure 7(b)

- detachment during single support phases, see the foregoing paragraph, and figure $7(\mathrm{c})$.

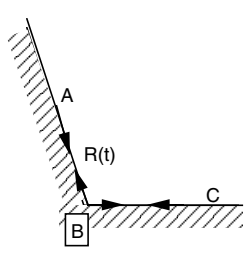

a)

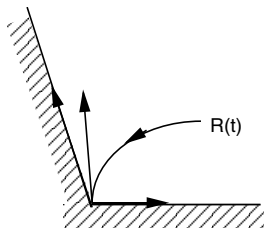

b)

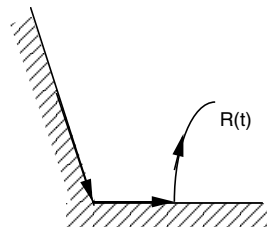

c)

Figure 7: Possible motions and shock outcomes in an abstract configuration space

The most recent studies in the field can be divided in three different approaches.

- The first one (Hurmuzlu \& Marghitu (1994); Han \& Gilmore (1993)) consists in an enumeration procedure. They apply a restitution law for

$\mathrm{RR} \mathrm{n}^{\circ} 4290$ 
simple impact at $A_{j}$ and sequentially propagate its effects on the other contact points $A_{i}, i \in \mathcal{I}(q)$. Nevertheless the overall process is assumed to be instantaneous, i.e. all shocks occur simultaneously at all the contact points : this is therefore really a multiple impact. At each point they investigate whether assuming zero or non-zero local percussions yield consistent outcomes. The main drawback of these studies is that they do not rule on whether or not the proposed algorithms always terminate with a unique solution. In the case of several admissible solutions, they don't give a criterion to make the choice between these solutions. However this should not be seen as a real drawback (except for simulation tool design purpose) since any heuristic to make the "good" choice, excluding in the same time several admissible solutions, drops the rigid body assumption.

- The second approach corresponds to the definition of a collision mapping

$$
\begin{aligned}
P_{c}: \partial \Phi \times\left\{-V\left(q\left(t_{k}\right)\right)\right\} & \longrightarrow \partial \Phi \times\left\{V\left(q\left(t_{k}\right)\right)\right\} \\
\left(q\left(t_{k}\right), \dot{q}\left(t_{k}^{-}\right)\right) & \longmapsto\left(q\left(t_{k}\right), \dot{q}\left(t_{k}^{+}\right)\right)
\end{aligned}
$$

where $V(q)=\left\{v \in \mathbb{R}^{p}: \forall i \in \mathcal{I}(q), v^{T} \nabla F_{i}(q) \geq 0\right\}$ denotes the tangent cone to $\partial \Phi$ at $q(t)$, i.e. the set of admissible post-impact velocities. In general one must assume that $V\left(q\left(t_{k}\right)\right)$ is not empty (Moreau (1985)). It is clear that the choice of $P_{c}$ should yield a mathematically, mechanically and numerically coherent formulation of the studied phenomenon. The so-called "sweeping or Moreau's process" (Brogliato (1999); Moreau (1985); Moreau (1988)) is a general formulation of the dynamics in (1)-(4) based on convex analysis tools. It allows one to write the dynamics as a particular Measure Differential Inclusion, a term coined by J. J. Moreau. It implicitly defines a collision mapping based on the computation of the post-impact motion via a proximation procedure in the kinetic metric

$$
\dot{q}\left(t_{k}^{+}\right)=\operatorname{prox}_{M\left(q\left(t_{k}\right)\right)}\left[\dot{q}\left(t_{k}^{-}\right), V\left(q\left(t_{k}\right)\right)\right]
$$

The main contributions to existential results relative to this approach are to be found in the book of Monteiro-Marques (Monteiro-Marques (1993)) and in (Mabrouk (1998)). They base on the use of a time discretization procedure similar to $(21)$; the discretized solutions are shown to converge 
to a function solution of $(\mathcal{S})$. For the moment they are restricted to the case $m=1$. See also (Paoli \& Schatzman (1993)) for related results : in particular existence of solutions (i.e. $q(t)$ absolutely continuous and $\left.\dot{q}(t) \in R C L B V^{4}\right)$ for the case $m>1$ and $T\left(t_{k}^{+}\right)=T\left(t_{k}^{-}\right)$has been proved in (Paoli (1993)), where $T(t)$ denotes the kinetic energy.

In (van der Schaft \& Schumacher (1996)) another collision mapping is introduced as follows : let $\mathcal{J}\left(q\left(t_{k}\right)\right)$ denote the set of constraints which were active before $t_{k}$ and will remain active in the near future, union the set of those which are going to be violated. $P_{c}$ is implicitly defined by the condition $\dot{q}\left(t_{k}^{+}\right)^{T} \nabla F_{j}\left(q\left(t_{k}\right)\right)=0, \forall j \in \mathcal{J}\left(q\left(t_{k}\right)\right)$. Both mappings are equivalent in the case of $\operatorname{card}\left(\mathcal{J}\left(q\left(t_{k}\right)\right)\right)=1$, but they do in general differ one from the other, as depicted in figures $8(\mathrm{a}),(\mathrm{b}),(\mathrm{c})$. Existence and uniqueness of solution to $(\mathcal{S})$ using this collision mapping has been proved for bimodal systems (the case $m=1$ ) and with no external actions ( $u=0$ and no gravity) in (van der Schaft \& Schumacher (1996)). We should note that both impact rules are dissipative. Although the particular impact rule introduced in (Moreau (1985); Moreau (1988)) makes it possible to prove well posedness for $m \geq 2$ (Heemels, Schumacher \& Weiland (1999)) the one in (van der Schaft \& Schumacher (1996)) does not facilitate such a proof.

Let us note that those mappings applied to the shock of two rigid bodies correspond to taking a zero restitution coefficient. Hence they may be named "generalized dissipative impact rules". However, it is possible to introduce some restitution $\delta \in[0,1]$ by substituting $\dot{q}\left(t_{k}^{+}\right)$in $(24)$ by $\frac{1}{2}(1+\delta) \dot{q}\left(t_{k}^{+}\right)+\frac{1}{2}(1-\delta) \dot{q}\left(t_{k}^{-}\right),($Mabrouk $(1998))$.

- The third approach (Lötstedt (1982)) can be seen as an extension of Gauss' principle (16) to the impact dynamics. The shock dynamics equation is given by

$$
M(q) \Delta \dot{q}=\nabla F(q) \Lambda_{n}
$$

where $\Delta \dot{q}=\dot{q}\left(t_{k}^{+}\right)-\dot{q}\left(t_{k}^{-}\right)$corresponds to the velocity jump at the instant of multiple impact $t=t_{k}$ and $\Lambda_{n}$ is the normal impulse.

Generalizing the complementarity relation (10) to the impulse level as

\footnotetext{
${ }^{4}$ Right Continuous with Local Bounded Variation
}

$\mathrm{RR} \mathrm{n}^{\circ} 4290$ 

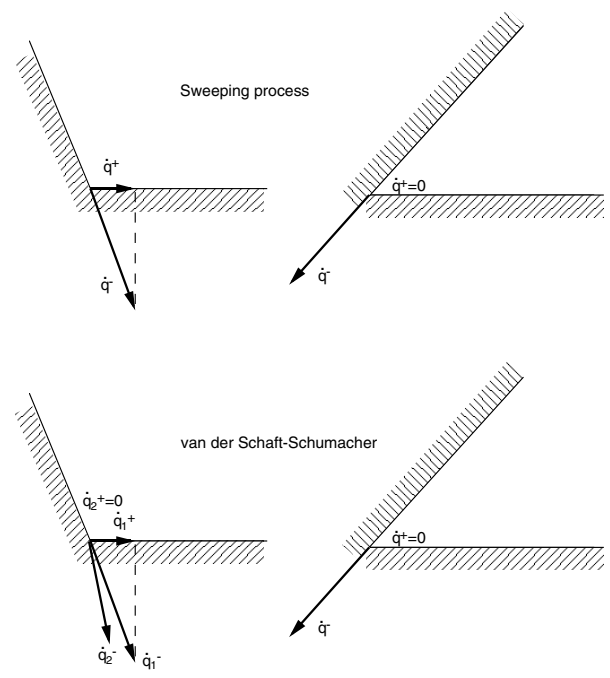

Figure 8: Collision mappings

follows

$$
\nabla F^{T} \dot{q}\left(t_{k}^{+}\right) \geq 0, \quad \Lambda_{n} \geq 0, \quad \Lambda_{n}^{T} \nabla F^{T} \dot{q}\left(t_{k}^{+}\right)=0
$$

and inserting the expression of $\dot{q}\left(t_{k}^{+}\right)$from (25) leads to the following LCP

$\nabla F^{T} \dot{q}\left(t_{k}^{+}\right)=\nabla F^{T} M^{-1} \nabla F \Lambda_{n}+\nabla F^{T} \dot{q}\left(t_{k}^{-}\right) \geq 0, \quad \Lambda_{n} \geq 0, \quad \Lambda_{n}^{T} \nabla F^{T} \dot{q}\left(t_{k}^{+}\right)=0$

Similarly to the continuous motion it can be shown that this LCP always possesses a solution $\Lambda_{n}$. Again $\nabla F \Lambda_{n}$ is unique.

The LCP in (27) is equivalent to the quadratic problem

$$
\min _{\dot{q}\left(t_{k}^{+}\right)} \frac{1}{2}\left(\dot{q}\left(t_{k}^{+}\right)-\dot{q}\left(t_{k}^{-}\right)\right)^{T} M\left(\dot{q}\left(t_{k}^{+}\right)-\dot{q}\left(t_{k}^{-}\right)\right), \quad \nabla F^{T} \dot{q}\left(t_{k}^{+}\right) \geq 0
$$

which can be seen as a generalized Gauss' principle. From (24) it is clear that as long as $V\left(q\left(t_{k}\right)\right)$ is a non-empty closed convex cone, (28) is equivalent to the sweeping process rule in (24) (Moreau (1988)). 
Let us mention that the classical decomposition of the impact phenomena into a phase of compression $\left[t_{A} \equiv t_{k}^{-}, t_{C}\right]$ followed by a phase of expansion $\left[t_{C}, t_{E} \equiv t_{k}^{+}\right]$with zero Poisson's coefficient, as done in (Pfeiffer \& Glocker (1996)), leads to the same results. The phase of compression can be formulated as in (27) by substituting $t_{k}^{-}, t_{k}^{+}$and $\Lambda_{n}$ by respectively $t_{A}, t_{C}$ and $\Lambda_{n C}$. Next using a similar substitution for the phase of expansion yields the following LCP

$\nabla F^{T} \dot{q}\left(t_{E}\right)=\nabla F^{T} M^{-1} \nabla F \Lambda_{n E}+\nabla F^{T} \dot{q}\left(t_{C}\right) \geq 0, \quad \Lambda_{n E} \geq 0, \quad \Lambda_{n E}^{T} \nabla F^{T} \dot{q}\left(t_{E}\right)=0$

with particular solution $\Lambda_{n E}=0$ leading to the unique solution $\dot{q}\left(t_{k}^{+}\right)=\dot{q}\left(t_{C}\right)$, for the same reason as above. Indeed the unique solution of the compression phase guarantees that $\nabla F^{T} \dot{q}\left(t_{C}\right) \geq 0$.

We illustrate the different approaches mentioned above on the 3-ball Newton's cradle, see figures 9 and 10. The initial data are $q_{2}(0)>q_{1}(0), q_{2}(0)=q_{3}(0)$ and $\dot{q}_{1}(0)=1, \dot{q}_{2}(0)=0, \dot{q}_{3}(0)=0$. In the configuration space, the system is sliding along the constraint $q_{2}(t)-q_{3}(t)=0$ and the multiple impact occurs at $t=t_{k}$. The kinetic energy loss is denoted as $T_{L}=T\left(t_{k}^{+}\right)-T\left(t_{k}^{-}\right)$.

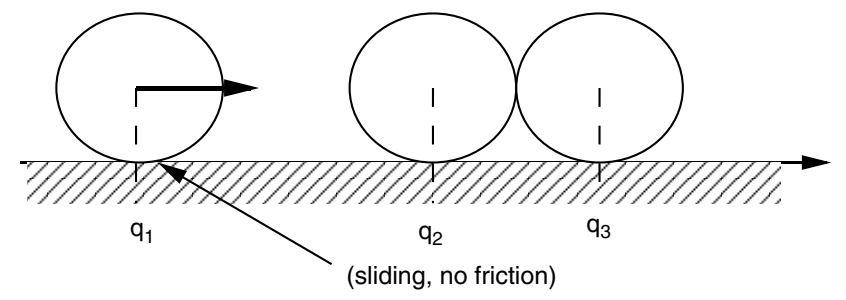

Figure 9: 3-ball system

\section{Comments}

1. In summary, well-posedness of $(\mathcal{S})$ depends on the well-posedness of the LCP, on the definition of a collision mapping (implicitly or explicitly), and on the applied forces $T u$ in (1). For instance it is shown in (Bressan $(1959))$ that non-uniqueness of the trajectory $(q, \dot{q})$ of $(\mathcal{S})$ can arise for certain external forces whereas the LCP in (13) has a unique solution.

$\mathrm{RR} \mathrm{n}^{\circ} 4290$ 


\begin{tabular}{c|c|c|c|c} 
& $\dot{q}_{1}\left(t_{k}^{+}\right)$ & $\dot{q}_{2}\left(t_{k}^{+}\right)$ & $\dot{q}_{3}\left(t_{k}^{+}\right)$ & $T_{L}$ \\
\hline (Han \& Gilmore (1993)) & $-1 / 3$ & $2 / 3$ & $2 / 3$ & 0 \\
\cline { 2 - 5 } & 0 & 0 & 1 & 0 \\
\hline (Pfeiffer \& Glocker (1996)) & $-1 / 3$ & $2 / 3$ & $2 / 3$ & 0 \\
\hline (van der Schaft \& Schumacher (1996)) & $1 / 3$ & $1 / 3$ & $1 / 3$ & $<0$ \\
\hline (Moreau (1988)) & $1 / 3$ & $1 / 3$ & $1 / 3$ & $<0$ \\
\cline { 2 - 5 } & $-1 / 3$ & $2 / 3$ & $2 / 3$ & 0 \\
\hline (Lötstedt (1984)) & $1 / 3$ & $1 / 3$ & $1 / 3$ & $<0$ \\
\hline (Haug, Wu \& Yang (1986)) & $-1 / 3$ & $2 / 3$ & $2 / 3$ & 0 \\
\cline { 2 - 5 } & $1 / 3$ & $1 / 3$ & $1 / 3$ & $<0$
\end{tabular}

Figure 10: Impact outcomes in the 3-ball system

On the contrary, as we remarked above, there are cases where the LCP possesses several solutions in terms of the contact forces, but $(\mathcal{S})$ possesses a unique solution $(q, \dot{q})$.

2. As long as well-posedness of $(\mathcal{S})$ is assured, one can choose any collision map. It is another problem to verify its practical usefulness.

3. All the proposed post-impact outcomes in figure 10 are a priori acceptable. The study of an approximating sequence of compliant problems, the contacts between the balls being modeled by linear springs with stiffnesses $k_{1}$ and $k_{2}$, shows that depending on the ratio $\alpha=\frac{k_{1}}{k_{2}}$ there is an infinity of possible outcomes. The two solutions in figure 10, $\dot{q}\left(t_{k}^{+}\right)=\left(-\frac{1}{3}, \frac{2}{3}, \frac{2}{3}\right)^{T}$ and $\dot{q}\left(t_{k}^{+}\right)=(0,0,1)^{T}$, correspond respectively to $\alpha=0$ and $\alpha=+\infty$, see (Brogliato (1999); Newby Jr. (1979); Ivanov (1995)).

4. Haug et al. (Haug, Wu \& Yang (1986)) deal with kinematic chains with a unique colliding point, at which Newton's conjecture is applied. It is supposed that all the active constraints remain active after the shock: in figure (7) this corresponds to trajectories from $A$ to $B$ and then from $B$ to $A$. Clearly this is not sufficient to model a walking machine. 
5. It is interesting to note that despite their discrepancies the above impact rules proposed in (Han \& Gilmore (1993); Pfeiffer \& Glocker (1996); Moreau (1988); Haug, Wu \& Yang (1986)) yield $\dot{q}\left(t_{k}^{+}\right)=\left(-\frac{1}{3}, \frac{2}{3}, \frac{2}{3}\right)^{T}$ as a solution, when $T_{L}=0$. It is noteworthy that the "classical" textbook solution is usually rather given by $\dot{q}\left(t_{k}^{+}\right)=(0,0,1)^{T}$, which is the postimpact motion if one assumes that $q_{2}\left(t_{k}\right)<q_{3}\left(t_{k}\right)$ and the restitution coefficient $e=1$ between the 3 balls (a succession of two simple inelastic shocks).

\subsection{Contacts with Amontons-Coulomb's friction}

In the frictionless case we saw that the LCP (13) was well-posed since the only possible indeterminacies, resulting from dependent active constraints, don't influence the global motion of the mechanical system (uniqueness of the acceleration of the center of mass). We will see in the sequel that the problem becomes more complicated through the introduction of dry friction, an essential parameter of the legged locomotion. In the part dedicated to the continuous motion, we will show that a decomposition of the tangential force/acceleration characteristic leads again to a LCP, whose unknown variables are related to the complete interaction forces.

\subsubsection{Continuous motion}

The first step consists in formulating the frictional law in a adequate way to determine the tangential generalized forces $P_{t}(q, \dot{q})$. Let us denote as $\dot{F}_{t, i}(q, \dot{q})=$ $G_{i}^{T}(q) \dot{q}, i \in \mathcal{I}(q)$ the relative tangential velocities at the contact points. Without any loss of generality, in the sequel we will focus on Coulomb's frictional law in the $2 \mathrm{D}$ case which classically states that

$$
\forall i \in \mathcal{I}(q):\left\{\begin{array}{l}
\dot{F}_{t, i}(q, \dot{q})=0 \Longrightarrow\left|\lambda_{t, i}\right| \leq \mu_{0, i} \lambda_{n, i} \\
\dot{F}_{t, i}(q, \dot{q})<0 \Longrightarrow \lambda_{t, i}=+\mu_{i} \lambda_{n, i} \\
\dot{F}_{t, i}(q, \dot{q})>0 \Longrightarrow \lambda_{t, i}=-\mu_{i} \lambda_{n, i}
\end{array}\right.
$$

where $\mu_{0, i}$ and $\mu_{i}$ denote the friction coefficients for stiction and sliding motion at the contact point $i$, and $P_{t}(q, \dot{q})=\Sigma_{i \in \mathcal{I}(q)} G_{i}(q) \lambda_{t, i}$. The basic idea is that

$\mathrm{RR} \mathrm{n}^{\circ} 4290$ 
the graph of the relay (or signum) characteristic can be expressed by a set of complementarity-slackness conditions. Setting

$$
\left\{\begin{array}{l}
\lambda_{t, i}^{+}=\mu_{0, i} \lambda_{n, i}+\lambda_{t, i} \\
\lambda_{t, i}^{-}=\mu_{0, i} \lambda_{n, i}-\lambda_{t, i} \\
\dot{F}_{t, i}^{+}=\frac{1}{2}\left(\left|\dot{F}_{t, i}\right|+\dot{F}_{t, i}\right) \\
\dot{F}_{t, i}^{-}=\frac{1}{2}\left(\left|\dot{F}_{t, i}\right|-\dot{F}_{t, i}\right)
\end{array}\right.
$$

the following complementarity relations express the friction model

$$
\forall i \in \mathcal{I}(q):\left\{\begin{array}{l}
\dot{F}_{t, i}^{+} \geq 0, \quad \lambda_{t, i}^{+} \geq 0, \quad \dot{F}_{t, i}^{+} \lambda_{t, i}^{+}=0 \\
\dot{F}_{t, i}^{-} \geq 0, \quad \lambda_{t, i}^{-} \geq 0, \quad \dot{F}_{t, i}^{-} \lambda_{t, i}^{-}=0
\end{array}\right.
$$

Let $\mathcal{I}_{0}(q, \dot{q}) \subset \mathcal{I}(q)$ be the set of potentially sticking contacts

$$
\mathcal{I}_{0}(q, \dot{q})=\left\{i \in \mathcal{I}(q) \mid \dot{F}_{t, i}(q, \dot{q})=0\right\}
$$

From (30) the tangential forces of the sliding contacts can be directly related to their relative normal forces by

$$
\forall i \in \mathcal{I}(q) \backslash \mathcal{I}_{0}(q, \dot{q}), \lambda_{t, i}=-\mu_{i} \lambda_{n, i} \operatorname{sign}\left(\dot{F}_{t, i}\right)
$$

Then since tangential relative velocities and accelerations have the same sign for transition from sticking to sliding, a complete model for the potentially sticking contact can expressed as

$$
\forall i \in \mathcal{I}_{0}(q, \dot{q})\left\{\begin{aligned}
\left|\lambda_{t, i}\right| & <\mu_{0, i} \lambda_{n, i} \Longrightarrow \ddot{F}_{t, i}=0 \\
\lambda_{t, i} & =+\mu_{0, i} \lambda_{n, i} \Longrightarrow \ddot{F}_{t, i} \leq 0 \\
\lambda_{t, i} & =-\mu_{0, i} \lambda_{n, i} \Longrightarrow \ddot{F}_{t, i} \geq 0
\end{aligned}\right.
$$

As shown in (Pfeiffer \& Glocker (1996); Trinkle, Pang, Sudarsky \& Lo (1995)), the conditions in (35) can also be replaced equivalently by a set of complementarityslackness conditions. This allows one to rewrite the dynamics in $(1),(2),(3)$ as

$$
M \ddot{q}=h+\left(W+N_{\text {slide }}\right) \lambda
$$




$$
\left\{\begin{array}{l}
y=A x+b \\
y \geq 0, \quad x \geq 0, \quad y^{T} x=0
\end{array}\right.
$$

for appropriate matrices $A$ and $b, W$ and $N_{\text {slide }}$, where the components of $x$ are suitable slack variables.

\section{Comments}

1. $3 D$ case

The above formulation can be extended to the 3D case by way of the decomposition of the tangential component of the force/acceleration at the rolling contact on two orthogonal directions $t$ and $o$ in the tangent plane. Coulomb's law stipulates that the contact force remains within or on the boundary of friction cone, that is

$$
\forall i \in \mathcal{I}(q), \quad \lambda_{t, i}^{2}+\lambda_{o, i}^{2} \leq \lambda_{n, i}^{2}
$$

These nonlinear constraints cause the model to become nonlinear, leading to a Nonlinear Complementarity Problem (NCP), see (Trinkle, Pang, Sudarsky \& Lo (1995); Stewart \& Trinkle (1996)). A way to overcome this difficulty consists in an approximation of the friction cones at the rolling contacts by four-sided friction pyramids, coming back to a LCP. The price to pay is, however, and increasing number of LCPs to be solved. The interested reader should refer to (Trinkle, Pang, Sudarsky \& Lo (1995)) for further details, and to (Pang \& Trinkle (1996)) for an extension to locally planar finite contact regions transmitting moments about the normals of the distributed contacts.

2. Existence and uniqueness of solutions to the $L C P$

As for every LCP formulation the problem of the existence of solutions is directly related to the positivity properties of the matrix $A$ in (37). $A$ is said to be a $\mathbf{P}_{0}$-matrix if all its principal submatrices have nonnegative determinants. $A$ is called a $\mathbf{P}$-matrix if all its principal submatrices have positive determinants. The main result relative to existence of solutions to general LCP states that, if $A$ a $\mathbf{P}$-matrix, then for every vector $b$, the LCP in (37) has a unique solution. Uniqueness of the solution is lost if $A$ is only a $\mathbf{P}_{0}$-matrix.

$\mathrm{RR} \mathrm{n}^{\circ} 4290$ 
It is noteworthy that in the frictionless case $\left(\mu_{0, i}=\mu_{i}=0, \forall i \in \mathcal{I}(q)\right)$, (37) reduces to (13) where $A$ is at least a $\mathbf{P}_{0}$-matrix (in the case of dependent active constraints). A very useful property of $\mathbf{P}$-matrices, respectively $\mathbf{P}_{0}$-matrices, lies in the fact that they are invariant under small perturbations. In other words, if $A$ is a $\mathbf{P}$-matrix, respectively $\mathbf{P}_{0^{-}}$ matrix, (which is the case in the frictionless motion), then all matrices $\tilde{A}$ sufficiently close to $A$ are also $\mathbf{P}$-matrices, respectively $\mathbf{P}_{0}$-matrices. In sight of this property, one should now intuitively understand the most advanced theoretical result we could find in the literature (Trinkle, Pang, Sudarsky \& Lo (1995)) : If the active constraints are independent, then there exists a positive scalar $\bar{\mu}$ such that if $\mu_{i} \in[0, \bar{\mu}], \forall i \in \mathcal{I}(q) \backslash \mathcal{I}_{0}(q, \dot{q})$, the $3 D$ contact problem with Coulomb cone or pyramidal approximation has a solution. In other words, if the friction coefficients at the sliding contacts are sufficiently small, then a solution to the LCP always exists. If $\mu_{i} \in[0, \bar{\mu}], \forall i \in \mathcal{I}(q)$, then uniqueness of the solution is also guaranteed. The main problem is now to find a correct estimate of the upper bound $\bar{\mu}$. The computation of such an estimate is nothing but trivial since $A$ is also a function of the state $(q, \dot{q})$. Further this estimate should not apply, since too small, to model real systems. This is probably due to the fact that the $\mathbf{P}$-property, respectively $\mathbf{P}_{0}$-property of $A$, on which the proof of the above given results bases, is a too strong assumption relative to existence of solutions to the LCP. It is only a sufficient condition, and one should try to relax it. In fact as said above, $\mathbf{P}$-property or $\mathbf{P}_{0}$-property of $A$ guarantees the existence of solution to (37), for every vector $b$. Turning to our advantage that $A$ and $b$ also depends on the state $(q, \dot{q})$, a practically interesting question to be answered to is : given $\mu_{i}$ with upper bound $\mu_{\max }=\max \left\{\mu_{i}, i=1,2, \ldots, m\right\}$ not necessary $\leq \bar{\mu}$ and starting from a consistent configuration at $t_{0}$, that is the LCP (37) admits a solution $\left(A\left(t_{0}\right)\right.$ is not necessary a $\mathbf{P}$ - or $\mathbf{P}_{0}$ matrix) how does the system, and thus $A$ and $b$, evolve? In other words, starting from a consistent configuration, does the system reach inconsistent configurations, if such configurations mathematically exist? To the best of our knowledge, this question, whenever practically crucial, seems not to have been intensively addressed until now and remains entirely open. One possible reason to that fact may lie in the underlying mathematical difficulties. 
The interested reader should refer to the more one century old benchmark Painlevé example and the new results relative to the above question in (Génot (1998); Génot, Brogliato, Brach \& Thuilot (1997)). Let us notice that the introduction of Amontons-Coulomb law at the tangential level increases the number of modes in which the system may evolve. Indeed from (32) each active contact adds 4 modes, where the one corresponding to $\dot{F}_{t, i}^{+}>0$ and $\dot{F}_{t, i}^{-}>0$ is impossible in view of (31).

3. One classical way to overcome the problem of existence and uniqueness of solutions to the LCP and to $(\mathcal{S})$ consists in the introduction of compliance at the contact points (Neimark (1995)) which in turn does not yield a suitable model for control design. Some authors also propose modifications of Coulomb's frictional law at the time-discretization level without founded physical motivation (Lötstedt (1984); Stewart \& Trinkle (1996)).

4. Notice however that for classical locomotion existence and uniqueness of the trajectory are assured as long as no foot-ground contact is sliding. Hence Painlevé paradoxes and indeterminacies do not exist during the course of normal walking.

\subsubsection{Multiple impacts}

The three different approaches described in the frictionless case in subsubsection 3.2.2 can be extended to the frictional case.

- The procedures in (Han \& Gilmore (1993); Han, Gilmore \& Ogot (1993b); Hurmuzlu \& Marghitu (1994)) are completed by the enumeration of the different sticking/sliding states at the contact points.

- Moreau proposes a formulation for a single purely inelastic shock in 2D in (Moreau (1988)), and for multiple impacts in (Moreau (1994)). The first algorithm in (Moreau (1988)) bases on an extension of AmontonsCoulomb's law at the impulse level, similar to the implicit algorithms of Whittaker (Whittaker (1904)) and Peres (Pérès (1953)), in which one supposes that the impulse direction is defined from the post-impact tangential velocity. The second algorithm in (Moreau (1994)) is part of a

$\mathrm{RR} \mathrm{n}^{\circ} 4290$ 
so-called complete contact law and handles multiple elastic shocks with restitution coefficients in the normal and tangential directions based on the introduction of the so-called average velocity at each contact. Particular attention is paid to the application to granular materials, i.e. to systems with many degrees of freedom $\left(\geq 10^{3}\right)$ and with simple continuous dynamics. Another impact rule for inelastic impact is obtained by stating conservation of certain angular momenta. This rule assumes no sliding to the contact points and is often used for biped robots (Kuo (1999)).

- Lötstedt in (Lötstedt (1984)) suggests an a priori "natural" generalization to the frictional case of Gauss' principle by rewriting the LCP in (27) as the following QP :

$$
\begin{aligned}
& \min \frac{1}{2} \Lambda^{T} H^{T} M^{-1} H \Lambda+\Lambda^{T} H^{T} \dot{q}\left(t_{k}^{-}\right) \\
& \Lambda_{n} \geq 0, \quad-\mu_{i} \Lambda_{n, i} \leq \Lambda_{t, i} \leq \mu_{i} \Lambda_{n, i}
\end{aligned}
$$

where $\Lambda=\left(\begin{array}{c}\Lambda_{n} \\ \Lambda_{t}\end{array}\right)$ and $H=\left(\begin{array}{ll}\nabla F & G\end{array}\right)$. However he shows that the Kuhn-Tucker conditions at the optimal point yield

$$
\Lambda^{T} H^{T} \dot{q}\left(t_{k}^{+}\right)=0
$$

Although this looks like an orthogonality relation between the impulse and the post-impact velocity, (40) with the fact that the impulse belongs to the friction cone does not imply any complementarity relation between the impulse and the post-impact velocity in the normal direction, which is the minimum requirement for a plastic shock. This means that in general such a rule can lead to rebounds. Moreover this rule is not energetically consistent in case of tangential velocity reversal. This approach has been applied to the modeling of feet ground impacts of bipedal robots (see (Fujimoto \& Kawamura (1998))).

Pfeiffer and Glocker in (Pfeiffer \& Glocker (1996))[Chapter 8] use Poisson's impact law (with compression and expansion phases). They extend Coulomb's friction law to the impulse level. The introduction of slackvariables similarly to the continuous motion allows them to formulate a 
LCP for each phase whose unknowns are the termination velocities and impulses. Modifications to the Poisson's restitution law and Coulomb's law for the expansion phase are introduced in order to cope with nonpenetration and energy consistency. In particular, since the matrix of the LCP for the compression phase has the same form as for the continuous motion, they cannot guarantee existence nor uniqueness of solutions.

- It is noteworthy that the above mentioned works (including Tzitzouris new law (Tzitzouris \& Pang (2000))) assure either existence of solutions at the cost of the physical consistency of the impact outcome or physically meaningful solutions at the cost of the existence and uniqueness property.

- Some authors study so-called tangential collisions, that is the behaviour of impact rules when the normal approach velocity tends to zero (Wang \& Mason (1992); Stronge (2000); Batlle (1993); Brach (1997)). One should notice that such grazing shocks and sliding phases analysis are quite different. Indeed in these studies the relative normal acceleration is strictly negative just before contact is made whereas all higher derivatives of the relative normal velocity are identically zero during a continuous sliding phase.

\subsection{Integration with numerical "time-stepping" algorithms}

From the foregoing sections it is clear that the complementarity relations between contact forces and relative positions play a crucial role in the overall dynamics of the system. This is even more true in the case of multiple contacts and multiple impacts. Consequently numerical integration schemes for such systems must incorporate all the nonsmooth and hybrid features of the dynamics. The reader is referred to (Brogliato, ten Dam, Paoli, Génot \& Abadie (2001)) for a complete survey on the numerical simulation of complementarity dynamical systems.

$\mathrm{RR} \mathrm{n}^{\circ} 4290$ 


\subsection{Summary of the motivations for using the rigid body complementarity framework}

- (36) (37) and (24) (26) (28) compactify in an elegant fashion all dynamics into a ODE + complementarity relations for monitoring detachments, impacts and dry friction, and set the basis for numerical simulations.

- When walking on most grounds, no one experiences any oscillation or motion of the feet due to sole or ground deformation (if any exists, it will remain completely neglectable compared to overall body motion). So modeling contacts by unilateral constraints is a realistic approximation.

- Main obstacles during integration of the dynamics due to coupling of friction and unilaterality are bypassed during walking since slippage is exceptionnal.

- Extensive comparisons of numerical and experimental results on circuit breakers (Abadie (2000)) have shown very good prediction capabilities (for systems more complex than bipeds, $m \approx 20$ vs. $m \approx 4$ ).

- Lumped flexibilities at joints can be inserted in the model.

- The approach benefits from lots of recent advances in mathematical, mechanical and numerical fields, a pre-requisite for stability and control study (see following section).

\subsection{Summary of existing work}

In the preceding sections we have presented a general framework for the study of mechanical systems with unilateral constraints. The main ingredients that enter the dynamics have been briefly reviewed. It is of interest to provide the reader with a general picture of the principal contributions to date. The works that we analyzed are those proposed by Baraff (Baraff (1993); Baraff (1994)), Lötstedt (Lötstedt (1982); Lötstedt (1984)), Hurmuzlu and Marghitu(Hurmuzlu \& Marghitu (1994); Marghitu \& Hurmuzlu (1995)), Trinkle et al. (Trinkle, Pang, Sudarsky \& Lo (1995)), Moreau (Moreau (1988); Monteiro-Marques (1993)), Pfeiffer and Glocker (Pfeiffer \& Glocker (1996)), Han and Gilmore 
(Han \& Gilmore (1993); Han, Gilmore \& Ogot (1993b)), Van der Schaft and Schumacher (van der Schaft \& Schumacher (1996)), Kunze and Neuman (Kunze \& Neumann (1997)), and Génot and Brogliato (Génot, Brogliato, Brach \& Thuilot (1997); Génot (1998); Génot \& Brogliato (1999)).

1. Friction

(a) Frictionless constraints (Lötstedt (1982); Baraff (1993); Baraff (1994); Trinkle, Pang, Sudarsky \& Lo (1995); Moreau (1988); Pfeiffer \& Glocker (1996); Han, Gilmore \& Ogot (1993b); van der Schaft \& Schumacher (1996))

(b) Static Amontons-Coulomb Friction (Baraff (1994); Trinkle, Pang, Sudarsky \& Lo (1995); Moreau (1988); Pfeiffer \& Glocker (1996); Han, Gilmore \& Ogot (1993b); van der Schaft \& Schumacher (1996))

(c) Dynamic Amontons-Coulomb Friction (Lötstedt (1984); Baraff (1993); Baraff (1994); Trinkle, Pang, Sudarsky \& Lo (1995); Moreau (1988); Pfeiffer \& Glocker (1996); Han, Gilmore \& Ogot (1993b); van der Schaft \& Schumacher (1996))

(d) 3-dimensional (Baraff (1994); Trinkle, Pang, Sudarsky \& Lo (1995))

2. Restitution rule for multiple impact (Lötstedt (1982); Lötstedt (1984); Moreau (1988); Pfeiffer \& Glocker (1996); Han \& Gilmore (1993); van der Schaft \& Schumacher (1996); Hurmuzlu \& Marghitu (1994); Tzitzouris \& Pang (2000))

3. Formulation with a L- or N-CP (Lötstedt (1982); Lötstedt (1984); Baraff (1993); Baraff (1994); Trinkle, Pang, Sudarsky \& Lo (1995); Moreau (1988); Pfeiffer \& Glocker (1996); Kunze \& Neumann (1997))

4. Analysis of inconsistencies (IW/OC) : no bounded solution to the LCP (Baraff (1993); Moreau (1988); Stewart \& Trinkle (1996); Génot, Brogliato, Brach \& Thuilot (1997); Génot \& Brogliato (1999))

5. Analysis of indeterminacies : several solutions to the LCP

(a) Indeterminacies due to friction (Lötstedt (1984); Baraff (1993))

RR $n^{\circ} 4290$ 
(b) Indeterminacies due to $\operatorname{rank} \nabla F<m$ (Lötstedt (1982))

6. Numerical simulation aspects

(a) Discretization of the dynamics procedure (Lötstedt (1984); Moreau (1988))

(b) Algorithm to solve the L- or N-CP

i. Lemke (Baraff (1993); Pfeiffer \& Glocker (1996))

ii. Specific (Lötstedt (1984); Baraff (1994); Trinkle, Pang, Sudarsky \& Lo (1995); Moreau (1986); Moreau (1988); Tzitzouris \& Pang (2000))

7. Existential problems

(a) LCP (Lötstedt (1982); Trinkle, Pang, Sudarsky \& Lo (1995); Stewart \& Trinkle (1996))

(b) NCP (Trinkle, Pang, Sudarsky \& Lo (1995))

(c) Well-posedness of $(\mathcal{S})$ (Monteiro-Marques (1993); van der Schaft \& Schumacher (1996); van der Schaft \& Schumacher (1996); Paoli \& Schatzman (1993); Schatzman (1978); Schatzman (1998); Percicale (1985); Percivale (1991))

8. Experimental validations of the models (Pfeiffer \& Glocker (1996); Abadie (2000))

\section{The stability framework}

The most crucial problem concerning the dynamics of bipedal robots is their stability. As has been explained in sections 2 and 3, a biped is far from being a simple set of (controlled) differential equations. Moreover the objectives of walking are quite specific. One is therefore led to first answer the question : what is a stable biped? And consequently what mathematical characterization of this stability can be constructed from the complementarity models ? As we will see next, this is closely related to the fact that bipeds can be considered as hybrid dynamical systems, the stability of which can be attacked 
from various angles. The goal of this section is to present some tools which can serve for the stability analysis of models as in (2) (3) (4) (5), and which are suitable for bipeds because they encapsulate their main features : occurrence of impacts, switching between types of motion, phases of continuous motion (manipulator-like behavior). Firstly we spend some time on describing invariant sets for complementarity systems. The point of view that is put forth is that various existing, or to be investigated, stability frameworks are better understood when invariant sets are classified. Secondly we review socalled impact Poincaré maps, which have been used extensively in the Applied Mathematics and Mechanical Engineering literature for vibro-impact systems (Masri \& Caughey (1966); Shaw \& Holmes (1983); Guckenheimer \& Holmes (1985); Shaw \& Shaw (1989); Babitsky (1998)). This point of view seems natural if one considers bipeds as jugglers as in (7). However it presents limitations which we point out.

\subsection{Invariant sets of systems subject to unilateral con- straints}

Application and use of mapping techniques is tightly coupled with the structure of the invariant set that represents the steady state motion. Systems subject to unilateral constraints behave in a more complex manner than the ones that are not (Budd \& Dux (1994)) (Ivanov (1993)). For example, let us consider the system given in Fig. (11.a). Suppose we would like to develop a controller to place the mass at a time varying position $x_{d}(t)=A+B \sin (\omega t)$ starting from an arbitrary initial condition in the admissible region. One can use a simple controller that yields the following closed loop dynamics:

$$
\begin{gathered}
m\left(\ddot{x}-\ddot{x}_{d}(t)\right)+k_{1}\left(\dot{x}-\dot{x}_{d}(t)\right)+k_{2}\left(x-x_{d}(t)\right)=\lambda_{n} \\
\text { subject to } \quad x \geq 0, \quad \dot{x}^{+}=-e \dot{x}^{-}, \quad \text { with } e \in[0,1)
\end{gathered}
$$

Our objective here is not to explore all possible types of invariant sets that may be attained by the system given by (41). Instead, we would like to show that the invariant sets of the closed loop system can be classified under three categories (see Fig. (11.b)):

i) Constraint Violating Invariant Sets (CVIS)

$\mathrm{RR} \mathrm{n}^{\circ} 4290$ 
An invariant set that includes at least one collision with the constraint surface per cycle of motion (including orbits that stabilize in finite time on the constraint surface after an infinite number of collisions). Hence an impact Poincaré mapping $P$ is well-defined that captures these orbits. This type of invariant set is a unique feature of systems subject to unilateral constraints. All the systems shown in Fig. (1) can be made to exhibit this behavior with a set of properly selected parameter values. Specifically, for locomotion systems, this will be the only mode of motion that can describe running and walking. The system in (41) may possess such invariant sets for proper choice of $x_{d}(t)$ and $k_{1}, k_{2}$.

ii) Unconstrained Invariant Sets (UIS)

An invariant set that does not include collisions with the constraint surface. In the single mass case, this corresponds to the cyclic motions of the mass that occur to the left of the constraint surface $(x>0 \quad \forall \bar{t} \leq$ $t \leq \infty)$. This type of invariant set can be observed for all systems of Fig. (1) except the juggler. For the biped, this corresponds to rocking when one or two limbs are in contact with the ground.

iii) Fully constrained Invariant Sets (FCIS)

An invariant set that never leaves the constraint surface. This corresponds for the system in (41) to a static equilibrium where the system rests on the constraint surface $(x(t)=0 \forall \bar{t} \leq t \leq \infty)$. All the systems in Fig. (1) can exhibit this behavior with a specific choice of the control parameter values and initial conditions.

Remark 2 The trajectory of a system may be both FCIS and UIS. For instance the dynamics of a manipulator subject to $m$ independent bilateral holonomic constraints can be written in a set of generalized coordinates $\left(x_{1}, x_{2}\right)$, $x_{1} \in \mathbb{R}^{m}$, such that the Lagrange equations are split into two parts (McClamroch 8 Wang (1988)): one part is $n-m$ dimensional and is the reduced order free-motion system, the other part is an algebraic relationship between the Lagrange multiplier and $x_{2}, \dot{x}_{2}, \ddot{x}_{2}$. Hence the trajectories are both UIS $\left(x_{2}, \dot{x}_{2}\right)$ and FCIS $\left(x_{1}, \dot{x}_{1}\right)$. Clearly if $m=n$ then the full system is constrained and the trajectory is FCIS. In certain cases similarly the trajectory will be both 
CVIS and UIS. Consider next a bipedal robot that rocks with one limb on the ground while the other swings freely, while striking a nail with a hammer in the right hand. The corresponding invariant set for the overall system may be classified as CVIS-UIS-FCIS. Similarly if it holds a fixed wall with the left hand. In any case, the control problem will be simplified by re-writing the dynamics in a proper set of generalized coordinates, taking into account which constraints are being part of the dynamical behaviour.

Existence of various types of motion leads to significant complexities in controlling the motion of systems with unilateral constraints. It is noteworthy that the above classification is not an artificial one, but does correspond to three quite different modes of complementary-slackness systems (Brogliato, Niculescu \& Monteiro-Marques (2000); Brogliato, Niculescu \& Orhant (1997)). Actually our point here is that the description of the modes has to rely on the nature of trajectories, and not only on the complementarity conditions. Hence according to this choice, the system in figure 11 is not bimodal but has three modes. In particular notice that although the complementarity relations in (41), i.e. $x \geq 0, \lambda_{n} \geq 0, x \lambda_{n}=0$ a priori define two modes $x>0$ and $x=0$ (hence a bimodal system (van der Schaft \& Schumacher (1998))) for control and dynamic systems analysis purpose one is led to consider those phases that correspond to CVIS as independent ones: they are described neither by the free motion nor by the constrained motion dynamics but by the whole dynamics of the hybrid system (the Lagrange equations, the impact rule and the complementarity relations). As a typical system whose trajectories are CVIS followed by FCIS, one may consider the classical bouncing ball whose dynamics when $x>0$ do not possess any fixed point, whereas the whole system does possess a globally stable equilibrium at $(x, \dot{x})=(0,0) \in$ FCIS. This example helps understanding the fundamental difference between complementary-slackness systems and systems of ODEs with impulsive disturbances (Bainov \& Simeonov (1989)). In this sense the system in (41) is equivalent to the bouncing ball when $x_{d}(t)$ takes both positive and negative values. The hybrid nature of such systems calls for new control approaches that can accommodate various types of motion, which may include discontinuous and uncontrollable phases such as the impact case. This will be discussed in the next subsection.

$\mathrm{RR} \mathrm{n}^{\circ} 4290$ 


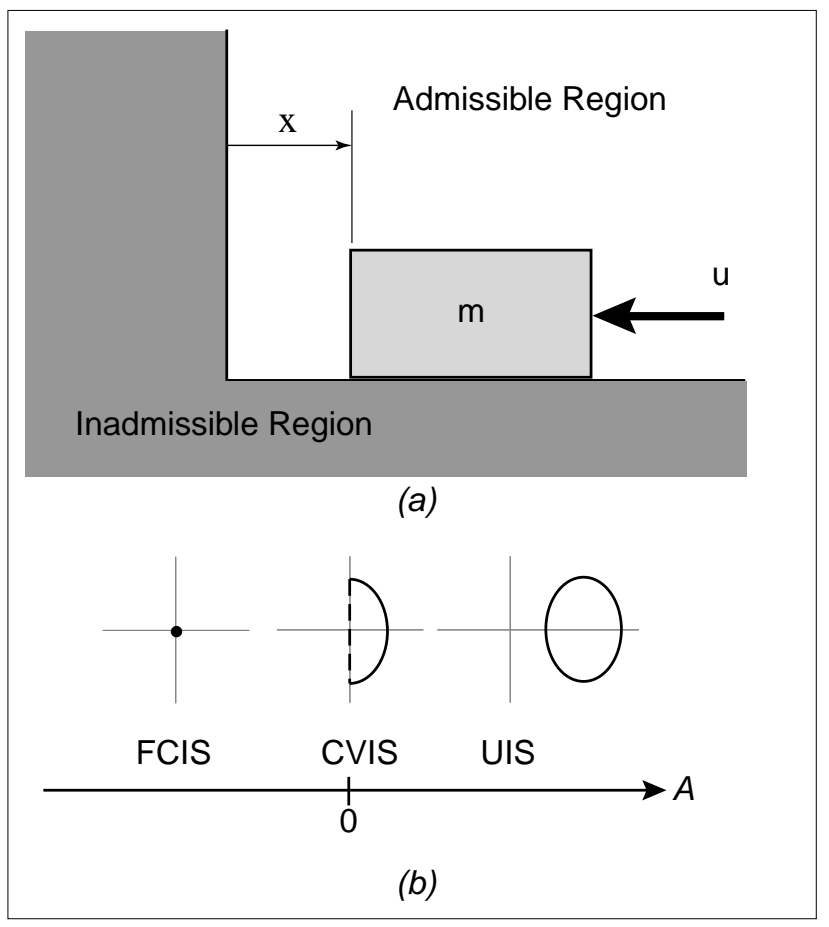

Figure 11: Controlled mass subject to a unilateral constraint

\subsection{Bipedal robots as hybrid dynamical systems}

The above classification of the invariant sets naturally leads one to consider complementary-slackness systems as in Fig. 1 as hybrid dynamical systems whose Discrete-Event-System (DES) states are defined from the described invariant sets. In the following we shall generically denote the phases that correspond to CVIS as $I_{k}$ and those that correspond to FCIS and/or UIS as $\Omega_{k}$. With some abuse of notation, we shall denote the DES states and the corresponding time intervals in the same manner. As we shall see further subdivisions will be needed. As an example let us consider an impact damper as in Fig. 1 (c) and with a sinusoidal excitation applied to the basis mass: for a proper choice of the spring-dashpot, the excitation parameters and of the initial conditions, the system possesses periodic trajectories with one impact 
per period (hence CVISs) (Masri \& Caughey (1966)). Thus one has

$$
\mathbb{R}^{+}=I_{0}
$$

Consider now a manipulator as in Fig. 1 (e) that performs a complete robotic task with a succession of free-motion and constrained motions phases, during which it is explicitly required to track desired motion and/or contact force ((Brogliato (1999); Brogliato, Niculescu \& Orhant (1997); Brogliato, Niculescu \& Monteiro-Marques (2000))). During the force/position control phases, the trajectories will in general be both UIS (in the tangent direction to the constraint surface) and FCIS (in the normal direction), see remark 2. During the free-motion phases the trajectories are UIS. It is therefore natural to split such task into three phases $\Omega_{2 k}, \Omega_{2 k+1}$ and $I_{k}$ that correspond to UIS, FCIS and CVIS respectively, i.e.

$$
\mathbb{R}^{+}=\Omega_{0} \cup I_{0} \cup \Omega_{1} \cup \Omega_{2} \cup I_{1} \cup \cdots
$$

Consider now the biped. It is clear that in order to describe walking, running and hopping motions, one needs more than the above three types of invariant sets. Moreover one needs more than the three phases proposed for the manipulator case. Indeed as we already pointed out concerning the choice of the Poincaré section, describing for instance a walking motion involves to take care of the non-sliding conditions. Hence one is led to differentiate contact phases (FCIS and UIS) and impact phases (CVIS and FCIS and/or UIS) that slide and those that stick. Notice moreover that this may be done independently of the presence of Amontons-Coulomb friction at the contact points: friction adds modes to the plant model, whereas our description concerns the nature of the trajectories and is directly related to stability and control objectives. But it is clear that the plant modeling will strongly influence the conditions under which those modes will be activated, see just below. Such a definition yields generally a large number of DES states. For a system with $m$ constraints, one gets

$$
\operatorname{card}\left(\mathcal{I}_{d}\right)=3^{m}+\sum_{i=1}^{m} 2^{i} 3^{m-i} C_{m}^{i}
$$

where $C_{m}^{i}=\frac{m !}{i !(m-i) !}$ and $\mathcal{I}_{d}$ is the DES state space. The formula in (44) may be found by inspection: for instance when there is no impact, then there may

$\mathrm{RR} \mathrm{n}^{\circ} 4290$ 
be either sliding or sticking contact, or detachment at any of the $m$ constraint surfaces, hence $3^{m}$ possible modes. In the case of a biped with $m=2$ (two feet and the rest of the constraints is not taken into account), one obtains 25 different modes. An enumeration of those 25 modes is useless here, so we shall define only those that are needed to describe the three mentioned types of motion:

- $\Omega_{k}^{f}$ : flight phases (both feet detached).

- $\Omega_{k}^{s l}$ : left foot sticks, right foot detached.

- $\Omega_{k}^{s r}$ : right foot sticks, left foot detached.

- $\Omega_{k}^{d s s}$ : double support phase, both feet stick.

- $I_{k}^{r s}$ : impacts on the right foot, left foot sticks.

- $I_{k}^{l d}$ : impacts on the left foot, right foot detached.

Then we obtain the following:

$$
\begin{array}{ll}
\text { Walking: } & \mathbb{R}^{+}=\Omega_{0}^{s l} \cup I_{0}^{r s} \cup \Omega_{0}^{d s s} \cup \Omega_{0}^{s r} \cup I_{0}^{r l} \cup \Omega_{1}^{d s s} \cup \Omega_{1}^{s l} \cup \cdots \\
\text { Hopping: } & \mathbb{R}^{+}=\Omega_{0}^{f} \cup I_{0}^{l d} \cup \Omega_{1}^{f} \cup I_{1}^{l d} \cup \cdots \\
\text { Running: } & \mathbb{R}^{+}=\Omega_{0}^{f} \cup I_{0}^{l d} \cup \Omega_{0}^{s l} \cup \Omega_{1}^{f} \cup I_{1}^{r d} \cup \Omega_{1}^{s r} \cup \Omega_{2}^{f} \cup I_{1}^{l d} \cup \cdots
\end{array}
$$

The basic idea is the following: the 25 different phases correspond to 25 DES states. One can construct a graph (grafcet, Petri net) that represents the DES. The conditions of activation of one mode have to be studied. For instance conditions such that sticking occurs at an impact or during a step have been studied in (Rubanovich \& Formal'sky (1981)), (Hurmuzlu (1993)), (Chang \& Hurmuzlu (1994)), and (Génot, Brogliato \& Hurmuzlu (1998)). They evidently strongly depend on the process model like Amontons-Coulomb friction and the multiple impact restitution law. The concatenation of phases in (45) corresponds to desired invariant sets of the DES (according to (Passino, Michel \& Antsaklis (1994)) there are two types of invariant sets in DES: either the system remains stuck in one state as in (42), or there is a cycle as the ones in (43) or (45)). From a general view point, any stability criterion should take into 
account both the nonsmooth and the hybrid natures of such complementaryslackness systems. As it is known, one may have several point of views of hybrid dynamical systems: continuous-time, discrete-event, or mixed, see e.g. (IEEE TAC Special Issue (1998); Automatica (1999)). It is not clear at this stage whether one of these manners to examine general hybrid dynamical systems applies better to complementary-slackness systems, and bipedal robots, than the others. Again, a lot will depend on the designer's and the task's goals. When a bipedal robot is required to strike a nail or to push an object, then the continuous-time level of stability and control will certainly supersedes the DES one. However when a general walking task is required, it is likely that one may not have to take care about accurate tracking of the legs and feet motion (some "crude" low-level controllers may be judged in this case to be sufficient). Then a supervisor-like control will be suitable: the robot will be required to track "a type of motion", which in more scientific terms will be translated into a DES invariant set. Clearly such a high-level control has to hinge upon some stability criterion that will enable the controller to keep the trajectories within some admissible set. The celebrated ZMP belongs to this category of criteria, and we shall come back on this in section 5.2.3. But one may not care about the swinging leg to perform its step with a strong accuracy. After all, human beings themselves each possess their own way to walk depending on their physical and physiological capabilities.

\subsection{Poincaré maps and stability}

Strong impacts may damage rigid structures and should therefore be avoided for walking robots. Nevertheless impact is part of the whole dynamics and cannot be neglected: when the robot is tracking a reference trajectory even with cautious foot placing, impact occurs since the orbit will remain only in a vicinity of the reference trajectory (even with high gains on the controller). From this intuitive consideration one could think that impacts act in a destabilizing manner. We argue at the opposite that impacts (which dissipate energy) play an important role in stabilizing the system.

Generally, the approach to the stability analysis takes into account two generally excepted facts about bipedal locomotion. The motion is discontinuous because of the impact of the limbs with the walking surface (Hurmuzlu

$\mathrm{RR} \mathrm{n}^{\circ} 4290$ 
\& Moskowitz (1987); Hurmuzlu (1993); Katoh \& Mori (1994); Zheng (1989); Grizzle, Abba \& Plestan (2001)). The dynamics is highly nonlinear and linearization about vertical stance generally should be avoided (Vukobratovic, Borovac, Surla \& Stokic (1990); Hurmuzlu (1993); Grizzle, Abba \& Plestan (1999)).

A classical technique to analyze dynamical systems was developed by the nineteenth century French mathematician Henry Poincaré. A formal mathematical description of the technique can be found in many previously published books and articles (Guckenheimer \& Holmes (1985); Parker \& Chua (1989)). Here we follow an informal presentation that describes the main features of the approach, its advantages, and its relevance in gait studies.

In the three-link bipedal model of section 2, we have shown that periodic motions of a simple biped can be represented as closed orbits in the phase space. Figure (4.d) depicts a first return map obtained from the points of the trajectory that coincide with the instant of heel strike. Poincaré map for a generalized coordinate $\phi_{1}$ (which is typically a joint angle in bipedal systems) at the instant of heel strike now can be obtained by plotting the values of $\phi_{1}$ at $i^{\text {th }}$ versus the values at $(i+1)^{\text {th }}$ heel strike. The iteration point that corresponds to the closed periodic orbit is on the $45^{\circ}$ degree line because when the motion is periodic $\phi_{1}$ at heel strike is identical for all successive locomotion steps. We also observe that points on the map accumulate at $p_{e}$ as the biped takes successive locomotion steps. The same construction can also be performed for the generalized velocity $\dot{\phi}_{1}$ or for any other kinematic quantity. One can equally construct a discrete map for the velocity of the center of mass of the upper member and investigate the evolution of locomotion speed with successive steps. Instead of choosing heel strike we could have chosen the instant of toe off for the present model and obtained similar plots.

In more general terms, the Poincare method is based on the construction of a first return map by considering the intersection of periodic orbits with an $n-1$ dimensional cross section in the $n$ dimensional state space. Let us mention a problem one has to face in the application of this method to bipedal locomotion when modeling the ground foot contacts by bilateral constraints. Splitting a locomotion cycle in a left foot support phase, a double support phase and a right support phase, a transition from one support phase to another one corresponds to removal and/or addition of kinematic constraints (Hemami \& 
Wyman (1979)). This problem can be seen as a two point boundary value problem where such transitions lead to changes in the dimension of the state space required to describe the dynamics and thus to changes in the vector of generalized coordinates. Notice however that this complication in the dynamics is of purely artificial nature, since it disappears in the unilateral constraint contact formulation, see section 3.2. In fact, the dimension of the state space remains constant $\left((q, \dot{q}) \in \mathbb{R}^{2 p}\right)$ and is independent of the support phase. The fact that the feet cannot penetrate into the ground only means that some region of the state space is unreachable.

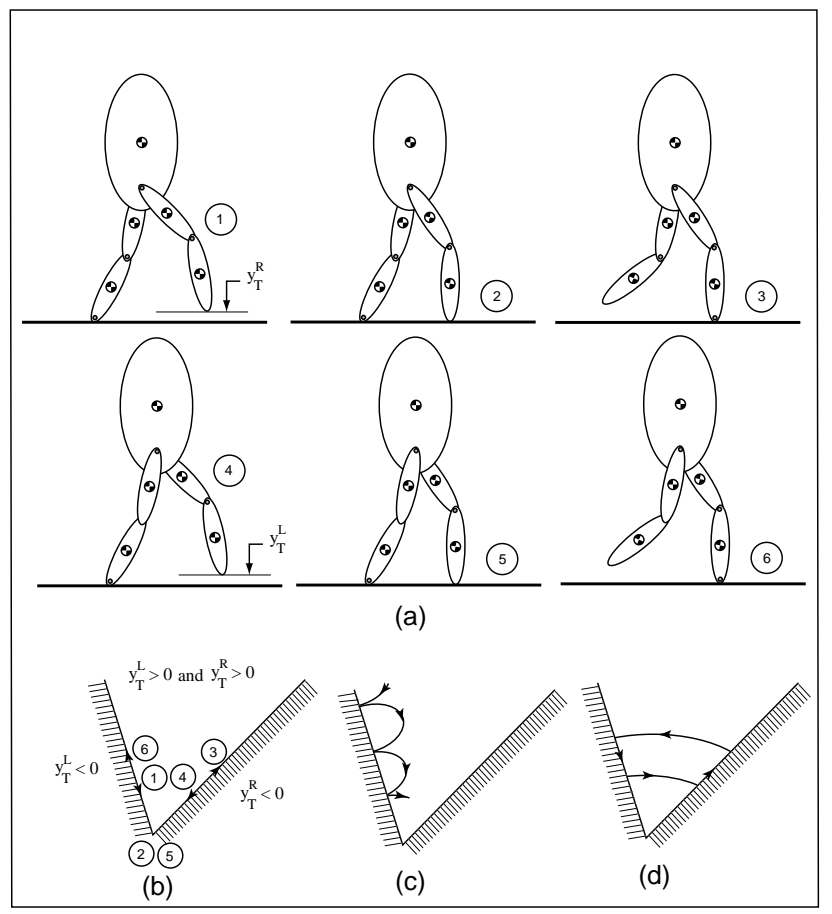

Figure 12: Walking, hopping and running

Due to the basic nature of discrete maps, the events that occur outside the cross section are ignored. For example, one can use simple sketches in the configuration space (see Fig. (12.a)) to represent the three motions that achieve progression in bipedal systems. Figures (12.b), (12.c), and (12.d) depict the representations of walking, hopping, and running respectively. We

$\mathrm{RR} \mathrm{n}^{\circ} 4290$ 
note that the depicted sketches are two dimensional projections that capture the essential dynamical features of higher dimensional bipedal systems. A simple choice of the Poincaré section may not lead to a mapping. For example, let us choose the section at the instant when the left foot contacts the walking surface $\left(y_{T}^{L}=0\right.$ in Fig. (12.a)). This section would not be crossed by trajectories that correspond to hopping on the right foot. Thus, we cannot define the map that corresponds to hopping on the right foot. One can choose an event such as the mere occurrence of heel strike, to define the Poincaré section (Hurmuzlu \& Moskowitz (1987); Kuo (1999)). This way, one can capture any of the three previously mentioned types of motion. Yet, even this choice would not lead to mappings for contact free rocking motions of the biped. This difficulty is not specific to bipedal system. The pendulum impact damper and the manipulator of Fig. (1) would also have this difficulty.

In addition, although such a selection would be sufficient for most impacting systems (see (Shaw \& Holmes (1983))), one needs to consider the slip conditions to develop mappings that describe dynamics of locomotion systems. Consider the walking motion and the corresponding family of orbits described by the sequence $1 \rightarrow 6$ in Fig. (12.b). The actual walking motions would be a subset of this family classified according to the slip conditions. One can construct several mappings depending on the type of motion. In general, however, the section can be written as follows:

$$
\Sigma_{i}^{+}=\left\{(q, \dot{q}) \in \mathbf{R}^{2 p} \mid t=t_{k}^{c}\right\} ; i=1, \ldots, l
$$

where the condition establishes the Poincaré section. In other words $y_{T}^{R}\left(t_{k}^{c}\right)=0$ or $y_{T}^{L}\left(t_{k}^{c}\right)=0$ (or both). In case there is an impact at the contacting instant (hence a velocity discontinuity) one may consider the right velocity $\dot{y}_{L}^{R}\left(t_{k}^{+}\right)$ and/or $\dot{y}_{T}^{L}\left(t_{k}^{+}\right)$to define the mapping (left velocities can be used as well). The subscript $i$ in (46) represents different motion types. The number $l$ depends on the types of contact with environment. For example consider hopping on a single limb, where there is a single contact point. For that case $l=2$, for bouncing with or without slippage. Accordingly, the two sections can be written as:

$$
\begin{aligned}
& \Sigma_{1}^{+}=\left\{(q, \dot{q}) \in \mathbf{R}^{2 p}\left|t=t_{k}^{c}\right| \dot{x}_{T}\left(t_{k}^{c,+}\right) \neq 0\right\} \\
& \Sigma_{2}^{+}=\left\{(q, \dot{q}) \in \mathbf{R}^{2 p}\left|t=t_{k}^{c}\right| \dot{x}_{T}\left(t_{k}^{c,+}\right)=0\right\}
\end{aligned}
$$


To be specific, there would be two types of contacts: instantaneous (rebound) or finite duration (contact is maintained for a while after the shock). In terms of the mapping, however, we will be only concerned with slippage conditions.

The discrete map obtained by following the procedure described above can be written in the following general form

$$
\xi_{i}=\mathbf{P}\left(\xi_{i-1}\right)
$$

where $\xi$ is a reduced dimension state vector, and the subscripts denote the $i^{\text {th }}$ and $i-1^{\text {st }}$ return values respectively.

Periodic motions of the biped correspond to the fixed points of $\mathbf{P}$ where

$$
\xi^{*}=\mathbf{P}^{k}\left(\xi^{*}\right)
$$

where $\mathbf{P}^{k}$ is the $k^{t h}$ iterate. The stability of $\mathbf{P}^{k}$ reflects the stability of the corresponding flow. Although, additional conditions are required to state that the Lyapunov stability of $\xi^{*}$ is equivalent to the Lyapunov stability of the overall trajectory of $(\mathcal{S})$. One of these, namely the continuous dependence of the trajectory on the initial conditions, was shown to be not verified on a simple biped model (Goswami, Thuilot \& Espiau (1996); Goswami, Thuilot \& Espiau (1997)) during passive gait. Another one is the continuity of the impact mapping which could also be questioned when accounting for dry friction; to the best of our knowledge, no regularization of Amontons-Coulomb's stickslip discontinuous model was proven to yield continuous impact mapping for all pre-impact states. Nevertheless, it seems reasonable to assume that these conditions are met in pratice for a wide subset of trajectories with an inelastic impact mapping.

The fixed point $\xi^{*}$ is said to be stable when the eigenvalues $\nu_{i}$, of the linearized map,

$$
\delta \xi_{i}=\mathbf{D P}^{k}\left(\xi^{*}\right) \delta \xi_{i-1}
$$

have moduli less that one. This technique employed in (Hurmuzlu \& Moskowitz (1987); Kuo (1999); Grizzle, Abba \& Plestan (1999); Grizzle, Abba \& Plestan (2001)) has several advantages. First, the stability of gait now conforms with the formal stability definition accepted in nonlinear mechanics. The eigenvalues of the linearized map (Floquet Multipliers) provide quantitative measures of the stability of bipedal gait. Finally, to apply the analysis to locomotion

$\mathrm{RR} \mathrm{n}^{\circ} 4290$ 
one only requires the kinematic data that represent all the relevant degrees

of freedom. No specific knowledge of the internal structure of the system is needed.

This feature also makes it possible to extend the analysis to the study of human gait. Using this approach one can develop quantitative measures for clinical evaluation of the human gait (Hurmuzlu \& Basdogan (1994); Hurmuzlu, Basdogan \& Stoianovici (1995)).

Let us recall that the straightforward application of Poincaré's method in order to identify stable periodic orbits requires the construction of a discretetime map from $\mathbb{R}^{n}$ to $\mathbb{R}^{n}$. Due to the nonlinear dynamics of biped robots, the construction of this map can only be achieved by numerical integration inducing a huge computational effort. This difficulty can be overcome for jumping robots by linearization of the continuous dynamics around a given trajectory (François \& Samson (1996)) and for some class of walking robots by reducing the dimension of the image of the Poincare map thanks to finite-time converging feedbacks applied on some selected part of the nonlinear dynamics (Grizzle, Abba \& Plestan (1999); Grizzle, Abba \& Plestan (2001)).

\section{Control of Bipedal Robots}

The control problem of bipedal robots can be defined as choosing a proper input $u$ in $(\mathcal{S})$ such that the system behaves in a desired fashion. The key issue of controlling the motion of bipeds still hinges on the specification of a desired motion. There are numerous ways that one can specify the desired behavior of a biped, which in itself is an open question. The control problem can become very simple or extremely complex depending on the specified desired behavior and the structure of the system. Typical bipedal machines are designed to perform tasks that are not confined to simple walking actions. Such tasks may include maneuvering in tight spaces, walking or jumping over obstacles, and running. In this article we place our main focus on tasks that are primarily related to walking. Therefore we will not be concerned with actions such as performing manipulation tasks with the upper limbs. 


\subsection{Passive Walking}

The idea of a biped walking without joint actuation during certain phases of locomotion was initially proposed Mochon and McMahon (Mochon \& McMahon (1980a)). The authors termed this type walk as "Ballistic Walking". The inspiration of this idea originated from evidence in human gait studies, which pointed out to relatively low levels of muscle activity in the swing limb during the swing period (see (Basmajian (1976)) and (Zarrugh (1976))). Two planar models were used: 1) a three element model with a single link stance and two link swing leg, 2) a four element model with two link stance and swing limbs. In each case, the authors searched for initial conditions at the onset of the swing phase such that the subsequent motion satisfied a set of kinetic and kinematic constraints. Then, they isolated the initial conditions that satisfied the imposed constraints. The simulation results were compared to experimentally measured knee angles and ground reaction forces. They concluded that their outcomes and human data had the same general shape. In (Mochon \& McMahon (1980b)) they improved their model by adding the stance knee (two element stance limb). With this more sophisticated model, the authors analyzed the model response by using three out of the five gait determinants (Saunders, Inman \& Eberhart (1953)).

The premise of walking without joint actuation, prompted McGeer (McGeer (1990)) to propose the "Passive Walking". McGeer developed numerical as well as experimental models of bipeds that have completely free joints. He demonstrated that these simple, unactuated bipeds can ambulate on downward planes only with the action of gravity.

Now, returning to (1), the typical passive control scheme is concerned with the free dynamics of the system $(\mathcal{S})$ given in section 3.1 (i.e. the dynamics of the system subject to $u=0$ ). Then, as we have shown in section 4.1 , a Poincaré section $\Sigma^{+}$can be selected (see (46)) to obtain a non-linear mapping in the form of:

$$
\xi_{i}=\mathbf{P}\left(\xi_{i-1}, \varphi\right)
$$

where the parameter vector $\varphi$ typically includes the slope of the walking surface, member lengths, and member weights. Then, the underlying question becomes the existence and stability of the fixed points of this map (Kuo (1999)) and the resemblance of the resulting motions to bipedal walking. This task

$\mathrm{RR} \mathrm{n}^{\circ} 4290$ 
is generally very difficult to realize with the exception of very simple systems. For example, in the case of vibro-impact systems, analytical expressions to show existence can be found ((Shaw \& Shaw (1989)) and (Babitsky (1998))). In the case of slightly more complex systems such explicit calculations become impossible since the free-motion dynamics are no longer integrable. Then one has to rely on numerical tools to derive both the Poincare mapping and its local stability (Kuo (1999)).

The main energy loss in these bipeds are due to the repetitive impacts of the feet with the ground surface. The gravitational potential energy provides the compensation for this loss, thus resulting in steady and stable locomotion for certain slopes and configurations. The ground impacts (see (Hurmuzlu \& Moskowitz (1986))) provide a unique mechanism that leads to stable progression in very simple bipeds as has been recently demonstrated by several investigators. In (Goswami, Thuilot \& Espiau (1996)) and (Goswami, Thuilot \& Espiau (1997)) the authors consider a simple model that includes two variable length members with lumped masses representing the upper body and the two limbs. The two members are connected at one end with a frictionless hinge. A third lumped mass is attached to this point, which represents the upper body. They analyze the nonlinear dynamics of (52) subject to prescribed variations in the elements of $\varphi$. They primarily focus on the effect of the ground slope, mass distribution, and limb lengths. Numerical analysis of the nonlinear map, results in the detection of stable limit cycles as well as chaotic trajectories that are reached through period doubling cascade.

A simpler, two link model was considered in (Garcia, Chatterjee, Ruina \& Coleman (1997)). The authors analyzed a biped that has the same structure as the one considered in (Goswami, Thuilot \& Espiau (1996)) but with fixed length limbs. The simplified model enabled the authors to develop an approximate analytical for the mapping $\mathbf{P}$. They used this map to analyze the dynamics of the motion subject to variations in the parameters (in their case they considered ground slope and mass ratios). They also demonstrated that this simple biped can produce stable locomotion as well as very complex chaotic motions reached through frequency doubling cascade.

The main challenge of the study of passive gait is to translate the understanding gained by studying passive systems to active systems. The MIT Leg Lab planar bipeds are controlled this way (Pratt (2000)) for periodic walking. 
In other words the desired trajectories $q_{d}(t)$ are designed from the study of passive walking. This is not the case for the Honda robots where $q_{d}(t)$ are obtained from human recordings. One of the main obstacles to build bipedal robots remains to be the prohibitively high joint torques that are often required to realize even routine walking tasks. A comprehensive investigation that bridges the passive studies to better design of active control schemes would be a natural extension of passive locomotion research.

\subsection{Walking with Active Control}

As we have seen in section 2 bipedal locomotion dynamics is a hybrid process that includes both smooth and discrete event dynamics. A properly designed control system can be effective in regulating the motion of the biped during smooth phases of motion that occur between successive feet ground impacts. On the other hand, in practice, one cannot inject direct control action into the impact process, which constitutes a major part of the discrete dynamics. This limitation makes the control of bipedal robots a challenging and unique process.

\subsubsection{Controller design}

The control action must assure that the motion of a multi-link kinematic chain, which can characterize a typical biped, is that of a walker. Although, the characteristics of the motion of a walker is still open to interpretation, we may translate this requirement to a set of target/objective functions given in the form:

$$
g_{i}\left(q_{d}(t), \dot{q}_{d}(t), \lambda_{n}, \lambda_{t}, \Theta, u\right)=0 \quad i=1, \ldots, k \leq p
$$

where $\Theta$ is a vector of parameters that prescribes certain aspects of the walking action such as progression speed, step length, etc.

Note that for the sake of simplicity of the notations $q$ will denote in the sequel the vector of generalized coordinates of the model considered by the referenced authors, i.e. either of the full order model, or of the reduced order model when assuming that the ground contacts, when active, are bilateral contacts.

$\mathrm{RR} \mathrm{n}^{\circ} 4290$ 
Investigators in the field developed a variety of control methods, ranging from simple linear controllers to sophisticated nonlinear schemes such as variable structure control and neural network control.

The control problem can be described as specifying the vector of joint actuator torques $u$ in (1) such that the system behaves in a certain way. The simplest way to proceed is to specify the time profiles of the joint trajectories. Investigators in the field used kinematics of human gait as desired profiles (see (Hemami \& Farnsworth (1977); Kho, Yurkovich \& Hemami (1987); Vukobratovic, Borovac, Surla \& Stokic (1990))). One can also simply specify certain aspects of locomotion such as walking speed, step length, upright torso, etc. (Chudinov (1980); Lavrovskii (1979); Lavrovskii (1980); Chudinov (1984); Beletskii (1975); Beletskii \& Kirsanova (1976); Beletskii \& Chudinov (1977b); Beletskii \& Chudinov (1980); Beletskii, Berbyuk \& Samsonov (1982); Grishin \& Formal'sky (1990); Novozhilov (1984); Hurmuzlu (1993); Chang \& Hurmuzlu (1994); Yang (1994)). In (Beletskii \& Chudinov (1980)), the authors use the components of the ground reaction forces in addition to kinematics in order to completely specify the control torques.

Once the objective functions are specified, one has to choose a control scheme in order to specify the joint moments (control torques) that drive the system toward the desired behavior. We encounter several approaches to this problem that can be enumerated as follows:

\section{Linear control}

The equations of motion are linearized about the vertical stance, assuming that the posture of the biped does not excessively deviate from this position. For example, in (Jalics, Hemami \& Clymer (1997); Kajita \& Tani (1996); Kajita, Yamaura \& Kobayashi (1992); Grishin, Formal'sky, Lensky \& Zhitomirsky (1994); Zheng \& Hemami (1984); Hemami, Zheng \& Hines; Golliday \& Hemami (1977); Hemami \& Farnsworth (1977); Gubina, Hemami \& McGhee (1974)) a PD controller in the form:

$$
u=K_{p}\left(q-q_{d}\right)+K_{d}\left(\dot{q}-\dot{q}_{d}\right)
$$

with $K_{p}$ and $K_{d}$ are positive definite gain matrices were defined to track joint trajectories. The linear controller, however, cannot track time functions. Thus, the authors discretized the desired joint profiles and let the controller track the trajectory in a point-to-point fashion. 


\section{Computed torque control}

When developing a computed torque algorithm, we define an error function as,

$$
\tilde{G}(q, \dot{q}, \ddot{q})=C_{1} \ddot{G}+C_{2} \dot{G}+C_{3} G=H(q) \ddot{q}+L(q, \dot{q}):=0
$$

where $G=\left\{g_{1}, \ldots, g_{k}\right\}^{T}, C_{1}, C_{2}$ and $C_{3}$ are $p \times k$ matrices containing the design parameters that will be explained below. If $g_{i}$ is a (virtual) holonomic constraint, then $i^{\text {th }}$ row of $C_{1}$ is set to $n_{i}$, whereas if the constraint is (virtual) non-holonomic, then the $i^{\text {th }}$ row of $C_{1}$ is set to 0 and the $i^{\text {th }}$ row of $C_{2}$ is set to $n_{i}$ where $n_{1}=\{1,0, \ldots, 0\}, n_{2}=\{0,1, \ldots, 0\}$ , etc. The matrices $C_{2}$ and $C_{3}$ are diagonal and contain parameters that can be chosen such that the solution set of $\tilde{G}$ is asymptotically stable about the origin (i.e. $g_{i} \rightarrow 0$ as $t \rightarrow \infty$ ).

Combining (1) and (55) yields $k$ relationships in the following form:

$$
H(q) M(q)^{-1} T u+H(q) M(q)^{-1}\left[-N(q, \dot{q})+\nabla F(q) \lambda_{n}+P_{t}(q, \dot{q})\right]+L(q, \dot{q})=0
$$

Thus, any control vector $u$ that satisfies (56) is assured to force state trajectories of the system to converge to the desired one. This method was applied (Hemami \& Katbab (1982); Lee \& Liao (1988); Hurmuzlu (1993); Yang (1994); Jalics, Hemami \& Clymer (1997)) to bipedal locomotion models with various level of complexities.

The computed torque method provides an efficient analysis tool of the system response subject to the objective functions. Yet, its use is practically limited because it works only when we have perfect knowledge of the system parameters.

\section{Variable structure control}

A nonlinear control method that resolves this problem is variable structure control (Bailey \& Arapostathis (1987); Slotine \& Li (1991)). This method results in a feedback law that ensures tracking despite uncertainties in system parameters. In this approach, one chooses the control vector as:

$$
u_{i}=\hat{u}_{i}-k_{i} \operatorname{sign}(s)
$$

$\mathrm{RR} \mathrm{n}^{\circ} 4290$ 
where $\hat{u}_{i}$ is the Slotine-Li controller with fixed estimated parameters. The second term, is the variable structure part of the control input, which insures robust tracking despite uncertainties in system parameters. The function $s$ defines the sliding surface that represents the desired motion. This is a high gain approach that is advantageous because it ensures convergence in finite time (we will come back later on the advantages of using finite-time convergent controllers). In locomotion, the stability of the overall motion relies on the effectiveness of the controller in eliminating the errors induced by impact during the subsequent step. The reader can check (Chang \& Hurmuzlu (1994)) to see the application of such a controller to a five-element planar model.

4. Optimal control

Optimal control methods have been used by researchers to regulate the smooth dynamic phase of bipedal locomotion systems. Two approaches have been taken to the optimization problem. The first method is based on computing the values of selected parameters in the objective functions that minimize energy based cost functions (Frank (1970); Vukobratovic (1976); Beletskii \& Chudinov (1977a); Beletskii, Berbyuk \& Samsonov (1982); Rutkovskii (1985); Channon, Hopkins \& Pham (1992b)). This approach cannot be characterized as optimal control according to the widely accepted terminology. The second approach is based on variational methods to obtain controllers that minimize cost functions (Beletskii \& Bolotin (1983); Bolotin (1984); Furusho \& Sano (1990); Channon, Hopkins \& Pham (1996a); Channon, Hopkins \& Pham (1996b)). It is the direct application of classical optimal control methods to bipedal locomotion, see (Channon, Hopkins \& Pham (1996c)) for the most advanced work in this topic. Here the authors regulate the motion of the biped over a support phase with a cost function of the following form:

$$
C_{o}=\int_{0}^{T}\left[\frac{1}{2} u u^{T}+p^{T}(f(q, u)-\dot{q})\right] d t+\lambda_{1}^{T}\left(h_{1}\left(q^{I}-q^{F}\right)+h_{2}\left(q^{I}\right)\right)
$$

where $f$ is the vector field of the reduced order dynamics corresponding to a motion with bilateral ground contact.

5. Adaptive control 
The adaptive control approach has received very little attention in biped control. Perhaps it is does not have real advantage in controlling bipedal locomotion. Nevertheless, Yang (Yang (1994)) has applied adaptive control approach to a three link, planar robot. It is clear that any adaptive technique that is suitable for robotic manipulators can be applied to the control of smooth dynamics phase of bipedal locomotion systems. Experiments have been led at the MIT Leg Lab (Pratt (2000)) using adaptive control.

\section{Shaping discrete event dynamics}

The abrupt nature of impact makes it practically impossible to directly control its effect on the system state. From a theoretical point of view, controlling the system during impacts would require the use of impulsive inputs $u$. This is clearly not possible both for mathematical and pratical reasons. Indeed the weel-posedness of systems as (1)-(4) with $u$ a Dirac measure is an open issue. Futhermore even an approximation of Dirac would demand actuators with too high bandwidth (to say nothing of induced vibrations in the mechanical structure). An alternate approach can be found in shaping the system state prior to the impact instant such that a desired outcome is assured.

Such an approach was taken in (Hurmuzlu (1993); Chang \& Hurmuzlu (1994)). In these studies, a set of objective functions in the form of (53) was tailored. Assuming perfect tracking, the authors derived the expression for the system state immediately before the instant of impact in terms of the parameter vector $\theta$. Subsequently, the post impact state was computed for specific values of the parameter vector. The parameter space was partitioned into regions according to slippage and contact conditions that result from the foot impact. Then, this partitioning was used to specify controller parameters such that the resulting gait pattern has only single support phase and the feet would not slip as a result of the feet impact. Dunn and Howe (Dunn \& Howe (1994)) developed conditions in terms of motion and structural parameters such that they minimize/eliminate the velocity jumps due to ground impact and limb switching. Thus, in their case, the objective of the shaping was to remove the effect of the impact altogether. Miura and Shimoyama (Miura 
\& Shimoyama (1984)) used a feedforward input that modifies the motion at the end of each step from measurements informations. Kuo (Kuo (1999)) proposed to stabilize an unstable (when uncontrolled) limit cycle created in a passive way on a 3D biped (a compass with a lateral degree of freedom that allows rocking side to side) by modifying the legs play during the steps.

7. Stability and periodic motions

Stability of the overall gait is often overlooked in locomotion studies. Typically, controllers have been developed, and few gait cycles have been shown to demonstrate that the biped "walks" with the given controller. A thorough analysis of the nonlinear dynamics of a planar, five element biped (Hurmuzlu (1993)) reveals a rich set of stable, periodic motions that do not necessarily conform to the classical period one locomotion. Tracking errors in the control action may lead to stable gait patterns that are different than the ones that are intended by the objective functions. One way to overcome this difficulty is to partition the parameter space such that one would choose specific values that lead to a desired gait pattern. This approach is taken in (Hurmuzlu (1993); Chang \& Hurmuzlu (1994))

\section{Other specialized control schemes}

Several investigators (Grishin \& Formal'sky (1990); Grishin, Formal'sky, Lensky \& Zhitomirsky (1994); Beletskii (1975); Chudinov (1984); Chudinov (1980); Katoh \& Mori (1994); Lavrovskii (1979); Lavrovskii (1980)) used simplified models without impact and constructed periodic trajectories by concatenation of orbits obtained from individually controlled segments of the gait cycle. This approach is quite similar in spirit to the Kobrinskii method (Kobrinskii (1965)) that is used the existence of trajectories of the impact damper and the impacting inverted pendulum (see Fig. (1)). (Blajer \& Schielen (1992)) compute a nonlinear feedforward torque corresponding to a "non-impacting" reference walk and use PD motion and PI force feedback to stabilize around the reference trajectory. Fuzzy logic control was used (Shih, Gruver \& Zhu (1991)) to develop a force controller that regulates ground reaction forces in sway- 
ing actions of an experimental biped. The authors were motivated by the fact that fuzzy logic methods can facilitate the development of advanced controllers to design bipeds that can walk on complex terrain. A group of investigators changed the parameters in the objective functions such that the desired motion is adapted to changing terrain conditions (Igarashi \& Nogai (1992); Shi \& Klein (1993); Zheng \& Sheng (1990)). Zheng (Zheng (1989)) used an acceleration compensation method to eliminate external disturbances from the motion of an experimental eight joint robot. He uses maximum available control action in order to eliminate the disturbances in minimum time. Kuo (Kuo (1999)) derives numerically an impact Poincaré map that represents the walking cycle, and proposes a linear state feedback that stabilizes this cycle. Clearly this is conceptually completely different from the works described above (see item (1) Linear control) since the design is based on a linearization of the Poincaré map itself and not of the continuous dynamics on one step. Grizzle et al. (Grizzle, Abba \& Plestan (1999); Grizzle, Abba \& Plestan (2001)) study a 2D 5-link biped with no ankle actuator. The system during contact phases is therefore underactuated. They propose an input/output linearization scheme and finite-time convergent algorithms which allow them to simplify the Poncaré map study, similarly to (Chang \& Hurmuzlu (1994); Zavala-Rio \& Brogliato (1999); Brogliato \& Zavala-Rio (2000)). The zero-dynamics is analyzed. Stability is tested numerically and the method is shown to be tractable (Grizzle, Abba \& Plestan (1999)). It is noticeable that the modeling approach they use corresponds to a particular dynamical regime of (1)-(4).

\subsubsection{DES stabilization}

Several studies fall in this category that is often based on a set of rules regulated by a high level strategies mostly inspired from biological systems. This approach is uniformly applied to regulate the locomotion of experimental robots. In practice one faces many difficulties that can be resolved by switching among several low level simple control schemes.

As we have shown in subsection 4.2 , walking corresponds to a particular sequence of activations of the modes of the Discrete Event System associated

$\operatorname{RR} n^{\circ} 4290$ 
to the biped seen as a complementary-slackness mechanical system. Such a sequence can be seen as an invariant set of the DES dynamics, see (45a). These control techniques aim at stabilizing this invariant set in the sense that the robot should ultimately be able to recover from falls and restart walking. Notice that this approach does not emphasize the low-level details of the walk (walking speed, steps length, etc.). An interesting approach in the area is the ZMP (Zero Moment Point) method proposed first by Vukobratovic and his co-workers ((Vukobratovic \& Juricic (1969); Vukobratovic, Borovac, Surla \& Stokic (1990))). The reader can refer to (Goswami (1999)) and references within for a detailed discussion regarding the real meaning of ZMP. Several different, but equivalent, definitions of the ZMP are given (Hemami \& Farnsworth (1977); Takanishi, Ishida, Yamaziki \& Kato (1985); Arakawa \& Fukuda (1997); Hirai, Hiros \& Kenada (1998)). The simplest one is (Hemami \& Farnsworth (1977)) the point where the vertical reaction force intersects the ground, i.e. the center of pressure. The ZMP stability criterion states that the biped will not fall down as long as the ZMP remains inside the convex hull of the foot-support. In these studies the authors impose the motion of the lower limb kinematics from human kinematic data, which they term synergies. This way, the ZMP criterion is used to switch between low-level controllers (which satisfy some objective functions like trajectory tracking), so as to stabilize the DES orbit in (45a) (in the sense of (Passino, Michel \& Antsaklis (1994))). The ZMP method was also applied with other controllers that are not based on prescribing human data (Borovac, Vukobratovic \& Surla (1989); Fukuda, Komota \& Arakawa (1997); Shi, Gruver \& Lee (1993)). One of the best example of the high degree of efficiency that such control approaches are able to attain are the bipeds constructed by Honda (Hirai, Hiros \& Kenada (1998); Hirai (1997); Pratt (2000)), whose control mainly rely on a suitable combination of local linear controller with high-level (or logical) conditions. In (Pratt, Chew, Torres, Dilworth \& Pratt (2001)) an intuitive approach for making some bipedal machines walk is proposed. It is based on so-called virtual model control, whose objective is to stabilize the DES orbit in (45a). 


\section{Conclusions and directions for future research}

This survey is devoted to the problem of modeling and control of a class of nonsmooth nonlinear mechanical systems, namely bipedal robots. It is proposed to recast these dynamical systems in the framework of mechanical systems subject to complementarity-slackness conditions. Unilateral constraints that represent possible detachment of the feet from the ground and Coulomb friction model can be written this way. Such a point of view possesses several advantages :

1. It provides a unified approach for mathematical, numerical and control investigations. This is a quite important point since numerical studies are mandatory in any mechanical and/or control design.

2. This framework encompassess all the models which have been used to study locomotion in the control and robotics literature.

3. Though we restrict ourselves to rigid body contact/impact models, lumped flexibilities can easily be introduced, both at the contact or in the structure itself (flexible joints). Introducing flexibilities may be necessary (Pratt (2000); Pratt \& Williamson (1995)). This will however make the control problem harder to solve.

4. Such models have proved to predict quite well the motion as several experimental validations available in the literature show.

5. As shown in this survey, the proposed modeling approach allows one to clarify which stability tools one may use to characterize the stability of a bipedal robot.

6. Finally it is the opinion of the authors that the only thing that is still missing in the field of bipeds design is a clear and general enough theoretical analysis framework, based on realistic models, that allows the designer to derive stable controllers taking into account the whole hybrid dynamics.

First, an overview of the main features of complementary-slackness mechanical systems is proposed, from the modeling and numerical analysis viewpoints.

$\operatorname{RR} n^{\circ} 4290$ 
Problems associated to multiple contact/impact without and with friction, and specific time discretization techniques - which are both of great importance in the field of bipeds control and design - are described in some detail. Second, it is proposed to cast such models in the framework of hybrid dynamical systems, i.e. an automaton connected with differential equations. The Discrete Event System (DES) part comes from the various modes in which the system evolves while walking, hopping or running. For instance walking corresponds to three modes (which constitute DES states): one foot in sticking contact with the ground (single support phases), or both feet in sticking contact (double support phase). The continuous part merely corresponds to the Lagrangian dynamics when the system evolves in one of the DES states - or modes -, which, except if both feet are detached from the ground, are viewed as bilaterally constrained subsystems. One advantage of such a modeling viewpoint is that it allows one to classify some stability analysis proposed in the literature: for instance certain controllers may only guarantee that the sequence of three modes for walking occurs in a robust manner, without taking care of accurate tracking of trajectories during the steps. Therefore they assure the stability of some invariant orbit of the DES part of the system. Other controllers may on the contrary focus on accurate tracking during the continuous phases, incorporating the impact : one may then speak of "low-level" stability. Within this context, impact Poincaré maps appear to be a powerful and suitable analytical tool to characterize the stability of a biped robot. Their use and the choice of Poincaré sections are discussed. In particular it seems that finite-time convergent controllers (e.g. dead-beat or sliding modes inputs) possess interesting features in this setting since they facilitate the characterization of closed-loop Poincaré maps, a task that is much harder with asymptotically stable schemes when one takes impact into account. Globally the dynamics and desired trajectories of bipeds that can walk place them somewhere in-between manipulators with unilateral constraints (Brogliato, Niculescu \& Orhant (1997); Brogliato, Niculescu \& Monteiro-Marques (2000)) and juggling systems (Zavala-Rio \& Brogliato (1999); Brogliato \& Zavala-Rio (2000)). How much has to be borrowed from each part and how much needs to be added to achieve stable walking remains largely open. Finally a review of various journal papers devoted to bipeds control published until recently is proposed. 
We also hope to develop technologies so that the humanoid robot can function not only as a machine, but blend in our social environment and interact with people, and play more important roles in our society (Hirai (1997)). To achieve this ambitious goal, future of the research in bipedal locomotion will be shaped by the completeness and complexity of the intended control system design. The degree of controller awareness that one wishes to incorporate in system design should guide the future of research in bipedal locomotion. For example, one may develop a controller assuming that the feet will never slip during locomotion. In contrast, if the control action ensures no slip walking, it must be aware of the friction constraint and possess the capability of preventing slippage (Génot, Brogliato \& Hurmuzlu (1998)). We may enumerate the controller awareness characteristics and their influence on future research as follows:

\section{Actuator awareness}

Control action should be tailored such that the control torques do not exceed a specified level actuator capacity. One of the main limiting factors in developing practical walking machines is the large capacity of actuators that are required to realize a particular control strategy. The answer seems to be in taking advantage of the structural configuration of the human biped in achieving minimal effort walking. Presently, research directed toward the better understanding of passive gait seems to be the main direction that is shaping future research to resolve the actuator problem. Studies that seek to develop active controllers that are based on dynamic principles of passive walk may provide the answer.

\section{Constraint awareness}

During locomotion, the biped is subject to unilateral constraints between its feet and the walking surface. Preservation or violation of these constraints significantly affects the dynamic characteristic of the gait and the resulting control system design. Feet detachments/attachments occur when the biped undergoes transitions among the single support, double supports, or airborne stages. Should the control action account for these transitions and include mechanisms to regulate them?

$\mathrm{RR} \mathrm{n}^{\circ} 4290$ 
This concerns the viability of the closed loop control (i.e. of the resulting orbit, see (Brogliato \& Zavala-Rio (2000)) for a definition of viability in this context). It is not only sufficient that the control brings the state toward a desired trajectory. It has to also assure that there are no unwanted impacts at the feet that may cause stumbling. Feet slippage is another important constraint violation that may take place during locomotion as well as at the instant of impacts. The control torques have to obey the inequality conditions (11) and (12) so that during the whole motion (smooth and nonsmooth): i) sticking is maintained, ii) detachment is monitored. It is clear that any control scheme has first to be shown to satisfy these two inequalities, otherwise cannot be declared to be stable, whatever other closed-loop properties it may possess. The inequalities in (11) and (12) can, in turn, be considered as conditions of transition between DES states and may be used as a measure of the robustness of the control scheme. Developing constraint aware controllers would be an important step toward developing bipedal robots that can handle unusual terrain conditions.

3. Terrain awareness

Should one use multiple control strategies that can be adapted and switched for various terrains? Or, should we design an extremely robust controller for even terrain that can also operate at more difficult terrain conditions? These questions remain to be answered, and their resolution may depend on how well we will understand the relationship among the control algorithm, stability, and the unilateral feet constraints.

4. Stability awareness Studying optimal trajectories, decreasing energy consumption, etc. on one step may certainly be useful, but represents only a fraction of what should be a complete approach. Moreover, suitability of controllers should be judged based on their performance over the hybrid dynamics. One may reach erroneous conclusions based on controller performance during the continuous phase only. A worse (in the sense of perhaps less robust, less suitable for adaptive control, less optimal, etc.) input may well lead to better locomotion stability than a better one. Bipeds considered as complementary slackness systems differ significantly (as dynamical systems) from smooth systems. Thus, their 
control may also require more original new methods and algorithms than the ones that have been developed for robotic manipulators. The on-line modification of the desired orbits $q_{d}(t)$ is a promising approach (Wieber (2000); Park \& Cho (2000)), which has already been applied experimentally (Hirai (1997); Hirai, Hiros \& Kenada (1998)). Also the design of $q_{d}(t)$ is in itself a challenge: it can be a consequence of passive walking analyses as for the MIT robots (Pratt (2000)) or be computed from human recordings (Honda's robots). The design of feedback controllers which stabilize the system around such orbits, whatever they may be, remains open when several steps (hence nonsmooth effects) are considered in the analysis, although significant progress in this direction has been made in (Grizzle, Abba \& Plestan (2001)). Another promising approach may be to extend Lyapunov's second method to nonsmooth complementarity mechanical systems, in order to design asymptotically stable low-level controllers. This has to incorporate all the nonsmooth features of walking, in particular impact phenomenon of the feet with the ground (by this we mean not only the impact phenomenon itself, but its consequence on the subsequent system, i.e. the creation of a new bilateral constraint - due to the complementarity relations - and its implication on stabilization). Such an approach has been taken in (Brogliato, Niculescu \& Orhant (1997); Brogliato, Niculescu \& Monteiro-Marques (2000)) for the case of fully actuated rigid manipulators subject to a single frictionless unilateral constraint. It is shown therein that a proper definition of the desired trajectories allows one to get asymptotic tracking (and the impacts play a major role in the stabilization process, since the kinetic energy loss they induce is wisely used in the Lyapunov function design). The case of bipeds evidently requires the extension of those works towards $m \geq 2$ constraints with friction. However due to the nature of impacts (purely inelastic) and the subsequent "reduced dimension" (see Figure 6) of the subspace in which the system evolves during a typical walking task (the authors in (Goodwine \& Burdick(2001)) speak of stratified configuration space for controllability studies), it is quite possible that the design may simplify. Clearly such a low-level tracking task would only constitute a module of a more complete controller, incorporating a supervising control (finite automaton) enabling the system to

$\mathrm{RR} \mathrm{n}^{\circ} 4290$ 
choose by itself a suitable trajectory to be tracked, and/or a suitable low-level controller with the required robustness capabilities.

Let us end this paper by recalling that a central issue in proving the stability of mechanical systems with several $(m \geq 2)$ unilateral constraints is that of discontinuity of orbits with respect to initial conditions (Brogliato (1999); Bressan (1959); Schatzman (1978)). However as depicted in Figure 6, the case of walking is quite specific in the sense that continuity with respect to initial data is likely to be verified for reasonable multiple impact laws as those presented in section 3.2.2 and implicitely assumed in (Grizzle, Abba \& Plestan (1999); Grizzle, Abba \& Plestan (2001)).

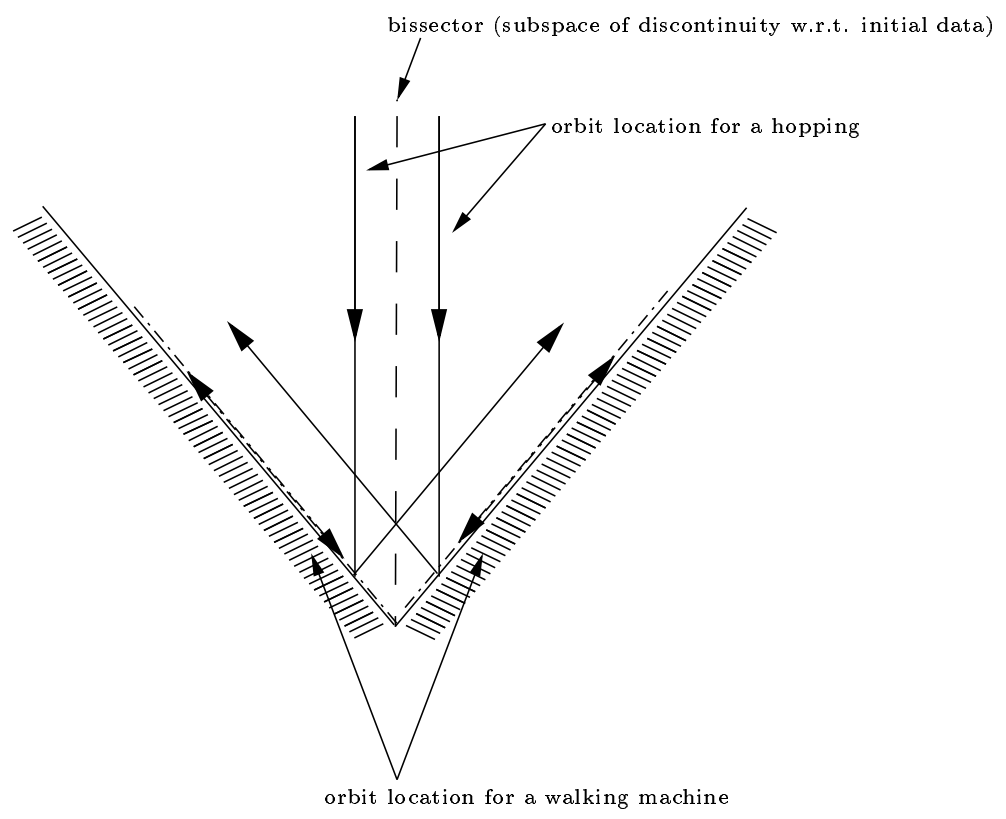

Figure 13: Discontinuity w.r.t initial conditions

This means that the study of a walking machine can be restricted to constraint surfaces of the configuration space (as advocated in (Goodwine \& Bur$\operatorname{dick}(2001))$ ) for a simpler class of switching systems), if one assumes that the constraints on the controller $u$ given in (11) and on the pre-impact state in 
(12) are satisfied. Consequently one promising direction for control design can be found in model predictive control strategies which enable to minimize some objective function while guaranteeing inequalities like (11) (12) and actuators limitations.

This paper has been written when the last author was with CNRS at the Laboratoire d'Automatique de Grenoble, France.

\section{References}

Abadie M. (2000). Dynamic simulation of rigid bodies : Modelling of frictional contact. Impacts in Mechanical Systems ; Analysis and modelling, Springer Lecture Notes in Physics LNP 551, B. Brogliato Ed., 61-144.

Anitescu M. \& Potra F. A. (1997). Formulating dynamic multi-rigid-body contact problems with friction as solvable linear complementarity problems. Nonlinear Dynamics, 14, 231-247.

Arakawa T. \& Fukuda T. (1997). Natural motion generation of a biped robot using the hierarchical trajectory generation method consisting of GA, EP layers. Proc. of the IEEE Conf. on Robot. and Automat., 1, Washington DC, 211-216.

Automatica (1999). A special Issue on Hybrid Systems, 35(3), March.

Babitsky V.I. (1998). Theory of Vibro-impact Systems and Applications. Springer Verlag, Foundations of Mechanical Engineering.

Bailey E. \& Arapostathis, A. (1987). Simple Sliding Mode Control Scheme Applied to Robot Manipulators. International journal of Control, 45(4), 1197-1209.

Bainov D. D. \& Simeonov P. S. (1989). Systems with impulse effects; stability, theory and applications. Ellis Horwood Series in Mathematics and its Applications, Wiley.

Baraff D. (1993). Issues in computing contact forces for non-penetrating rigid bodies. Algorithmica, 10, 292-352.

Baraff D. (1994). Fast contact force computation for nonpenetrating rigid bodies. SIGGRAPH'94, Orlando, July, 23-34.

$\mathrm{RR} \mathrm{n}^{\circ} 4290$ 
Basmajian J. V. (1976). The Human Bicycle. Biomechanics V-A, Komi P. V., Ed., University Park Press, 297-302.

Batlle J. A. (1993). On Newton's and Poisson's rules of percussive dynamics. ASME J. of Applied Mechanics, 60, 376-381.

Beletskii V. V. (1975). Dynamics of two legged walking, II. izv. AN SSSR. Mekhanika Tverdogo Tela, 10(4), 3-13.

Beletskii V. V., Berbyuk V. E. \& Samsonov V. A. (1982). Parametric optimization of motions of a bipedal walking robot. izv. AN SSSR. Mekhanika Tverdogo Tela, 17(1), 28-40.

Beletskii V. V. \& Bolotin Y. V. (1983). Model estimation of the energetics of bipedal walking and running. Mechanics of Solids, 18(4), 87-92.

Beletskii V. V. \& Chudinov P. S. (1977). Parametric optimization in the problem of bipedal locomotion. izv. AN SSSR. Mekhanika Tverdogo Tela, 12(1), 25-35.

Beletskii V. V. \& Chudinov P. S. (1977). The linear stabilization problem for two legged ambulation. izv. AN SSSR. Mekhanika Tverdogo Tela, 12(6), 65-74.

Beletskii V. V. \& Chudinov P. S. (1980). Control of motion of a bipedal walking robot. izv. AN SSSR. Mekhanika Tverdogo Tela, 15(3), 30-38.

Beletskii V. V. \& Kirsanova T. S. (1976). Plane linear models of biped locomotion. izv. AN SSSR. Mekhanika Tverdogo Tela, 11(4), 51-62.

Blajer W. \& Schielen W. (1992). Walking without impacts as a motion/force control problem. ASME J. of Dynamic Systems, Measurement and Control, 114, 660665 , December.

Bolotin Y. V. (1984). Energetically optimal gaits of a bipedal walking robot. $M e-$ chanics of Solids, 19(6), 44-51.

Borovac B., Vukobratovic M. \& Surla D. (1989). An Approach to Biped Control Synthesis. Robotica, 7, 231-241.

Brach R. M. (1991). Mechanical Impact Dynamics, John Wiley, New York.

Brach R. M. (1997). Impact coefficients and tangential impacts. ASME J. of Applied Mechanics, 34(4), 1014-1016. 
Bressan A. (1959). Incompatibilità dei teoremi di esistenza e di unicità del moto per un tipo molto comune e regolare di sistemi meccanici. Rend. Scu. Norm. di Pisa, 333-348.

Brogliato B. (1999). Nonsmooth Mechanics, Springer Verlag, CCES. Erratum and addendum at http://www-lag.ensieg.inpg.fr/publications.html.

Brogliato B., Niculescu S. I. \& Orhant P., (1997). On the control of finite dimensional mechanical systems with unilateral constraints. IEEE Trans. Automatic Control, 42(2), 200-215.

Brogliato B., Niculescu S. I. \& Monteiro-Marques M. (2000). On the tracking control of a class of complementarity-slackness hybrid mechanical systems. Systems and Control Letters, 39, 255-266.

Brogliato B., ten Dam A. A. , Paoli L., Génot F. \& Abadie M. (2001). Numerical simulation of finite dimensional multibody nonsmooth mechanical systems. ASME Applied Mechanics Reviews.

Brogliato B. \& Zavala-Rio A. (2000). On the control of complementary slackness juggling mechanical systems. IEEE Trans. Automatic Control, 45(2), 235-246.

Budd C. \& Dux F. (1994). Chattering and related behaviour in impact oscillators. Proc. R. Soc. London, A, 347, 365-389.

Chang T. H. \& Hurmuzlu Y. (1994). Sliding Control without Reaching Phase and its Application to Bipedal Locomotion. ASME Journal of Dynamic Systems, Measurement, and Control, 105, 447-455.

Channon P. H., Hopkins S. H. \& Pham D. T. (1992). Modelling and control of a bipedal robot. Journal of Systems Engineering, 2, 46-59.

Channon P. H., Hopkins S. H. \& Pham D. T. (1992). Derivation of optimal walking motions for a bipedal walking robot. Robotica, 10, 165-172.

Channon P. H., Hopkins S. H. \& Pham D. T. (1996). A gravity compensation technique for an n-legged robot. Proc. INSTN MECH ENGRS, ImechE, 210, 1-14.

Channon P. H., Hopkins S. H. \& Pham D. T. (1996). Optimal Control of an n-legged robot. Journal of Systems and Control Engineering, 210, 51-63.

$\mathrm{RR} \mathrm{n}^{\circ} 4290$ 
Channon P. H., Hopkins S. H. \& Pham D. T. (1996). A variational approach to the optimization of gait for a bipedal robot. Proc. INSTN MECH ENGRS, ImechE, 210, 177-186.

Chevallereau C., Formal'sky A. \& Perrin B. (1997). Control of a walking robot with feet following a reference trajectory derived from ballistic motion. IEEE Conf. Robotics and Automation, 20-25 April, Albuquerque, New Mexico, USA.

Chudinov P. S. (1980). One problem of angular stabilization of bipedal locomotion. izv. AN SSSR. Mekhanika Tverdogo Tela, 15(6), 49-54.

Chudinov P. S. (1984). Problem of angular stabilization of bipedal locomotion. $i z v$. AN SSSR. Mekhanika Tverdogo Tela, 19(1), 166-169.

Cottle R. W. \& Dantzig G. B. (1968). Complementary pivot theory of mathematical programming. Linear Algebra and its Applications, 1, 103-125.

Cottle R. W., Pang J. S. \& Stone R.E. (1992). The Linear Complementarity Problem, Academic Press, Boston, Massachusetts.

Delassus E. (1917). Mémoire sur la théorie des liaisons finies unilatérales. Ann. Sci. Ecole Normale Sup., 34, 95-179.

Dorn W. S. (1960). Duality in quadratic programming. Quaterly of Applied Mathematics, 18(2), 155-162.

Dunn E. \& Howe R. D. (1994). Toward Smooth Bipedal Walking. IEEE Int. Conf. on Robotics and Automation, 3, San Diego, CA, 2489-2494.

Dupont P. E. (1992). The effect of Coulomb friction on the existence and uniqueness of the forward dynamics problem. IEEE Int. Conf. on Robotics and Automation, Nice, France, May, 1442-1447.

Erdmann M. (1994). On a representation of friction in configuration space. Int. Journal of Robotics Research, 13(3), June, 240-271.

Ferris M. C. \& Pang J. S. (1997). Engineering and Economic Application of Complementarity Problems. SIAM Review, 39(6), 669-713.

Fischer A. (1992). A special Newton-type optimization method. Optimization, 24, 269-284. 
François C. \& Samson C. (1996). Energy efficition control of running legged robots. A case study: the planar one-legged hopper. INRIA Research Report, 3027, http://www.inria.fr/RRRT/RR-3027.html.

Frank A. (1970). An Approach to the Dynamic Analysis and Synthesis of Biped Locomotion Machines. Medical and Biological Engineering, 8, 465-476.

Fujimoto Y. \& Kawamura A. (1998). Simulation of an autonomous biped walking robot including environmental force interaction. IEEE Robotics and Automation Magazine, 5(2), 33-42.

Fukuda T., Komota Y. \& Arakawa T. (1997). Stabilization Control of Biped Locomotion Robot based Learning with Gas having Self-adaptive Mutation and Recurrent Neural Networks. IEEE Conf. Robotics and Automation, Albuquerque, New Mexico, USA, 217-222.

Furusho J. \& Sano A. (1990). Sensor based control of a nine link biped. International Journal of Robotics Research, 9(2), 83-98.

Garcia M., Chatterjee A., Ruina A. \& Coleman M. (1997). The Simplest Walking Model: Stability, and Scaling. ASME Journal of Biomechanical Engineering, 120, 281-288.

Génot F. (1998). Contributions à la modélisation et à la commande des systèmes mécaniques de corps rigides avec contraintes unilatérales. $\mathrm{PhD}$ Thesis, Institut National Polytechnique de Grenoble, France, February, http://www.inrialpes.fr/bip.

Génot F. \& Brogliato B. (1999). New Results on Painlevé paradoxes. European Journal of Mechanics A/Solids, 18(4), 653-677.

Génot F., Brogliato B., Brach R. M. \& Thuilot B. (1997). On LCPs and tangential impacts in rigid body mechanics with unilateral constraints and dry friction. IFAC Symposium on Robot Control (SYROCO'97), 3-5 September, Nantes, France.

Génot F., Brogliato B. \& Hurmuzlu Y. (1998). Control of sticking phases in mechanical systems with unilateral constraints. 7th Conf. on Nonlinear Vibrations, Stability, and Dynamics of Structures, July 26-30, Virginia Polytechnic Inst., Blacksburg, USA.

$\mathrm{RR} \mathrm{n}^{\circ} 4290$ 
Golliday C. L. \& Hemami H. (1977). An approach to analyzing biped locomotion dynamics and designing robot locomotion control. IEEE Transactions on Automatic Control, 22(6), 963-972.

Goodwine B. \& Burdick J. W. (2001). Controllability of kinematic control systems on stratified configuration spaces. IEEE Trans. Automat. Control 46(3), 358-368.

Goswami A. (1999). Postural Stability of Biped Robots and the Foot-Rotation Indicator (FRI) Point. International Journal Of Robotics Research, 18(6), 523-533.

Goswami A., Thuilot B. \& Espiau B. (1996). Compass Like Bipedal Robot Part I: Stability and Bifurcation of Passive Gaits. INRIA Research Report, 2996, http://www.inria.fr/RRRT/RR-2996.html.

Goswami A., Thuilot B. \& Espiau B. (1998). A study of the passive gait of a compasslike biped robot: symmetry and chaos. International Journal of Robotic Research, 17(12), 1282-1301.

Grishin A. A. \& Formal'sky A. M. (1990). Control of bipedal walking robot by means of impulses of finite amplitude. izv. AN SSSR. Mekhanika Tverdogo Tela, 25(2), $67-74$.

Grishin A. A., Formal'sky A. M., Lensky A. V. \& Zhitomirsky S. V. (1994). Dynamic of a vehicle with two telescopic legs controlled by two drives. The International Journal of Robotics Research, 13(2), 137-147.

Grizzle J.W., Abba G. \& Plestan F. (1999). Proving asymptotic stability of a walking cycle for a five dof biped robot model. 2nd Int. Conf. Climbingand Walking Robots, Portsmouth, UK, september, 69-81.

Grizzle J.W., Abba G. \& Plestan F. (2001). Asymptotically stable walking for biped robots: Analysis via systems with impulse effects. IEEE Trans. on Automatic Control, 46(1), 51-64.

Gubina F., Hemami H. \& McGhee R. B. (1974). On the dynamic stability of biped locomotion. IEEE Transactions on Biomedical Engineering, 21(2), 102-108.

Guckenheimer J. \& Holmes P. (1985). Nonlinear Oscillations, dynamical systems, and bifurcations of vector fields, Springer-Verlag, New York. 
Han I. \& Gilmore B. J. (1993). Multi-body impact motion with friction - Analysis, simulation and experimental validation. ASME J. of Mechanical Design, 115, September, 412-422.

Han I., Gilmore B. J. \& Ogot M. M. (1993). The incorporation of arc boundaries and stick-slip friction in a rule-based simulation algorithm for dynamic mechanical systems with changing topologies. ASME J. of Mechanical Design, 115, September, 423-434.

Haug E. J., Wu S. C. \& Yang S. M. (1986). Dynamics of mechanical systems with Coulomb friction, stiction, impact and constraint addition-deletion. Mechanisms and Machine Theory, 21(5), 401-406.

Hemami H. \& Farnsworth R. L. (1977). Postural and gait stability of a planar five link biped by simulation. IEEE Transaction on Automatic Control, 22, 452-458.

Hemami H. \& Katbab A. (1982). Constrained Inverted Pendulum Model for Evaluating Upright Postural Stability. ASME Journal of Dynamics Systems, Measurement, and Control, 104, 343-349.

Hemami H. \& Wyman B. F. (1979). Modeling and Control of Constrained Dynamic Systems with Application to Biped Locomotion in the Frontal Plane. IEEE Transaction on Automatic Control, 24(4), 526-535.

Hemami H., Zheng Y. F. (1984). Dynamics and control of motion on the ground and in the air with application to biped robot. J. Robotic Systems, 1(1), 101-116.

Hemami H., Zheng Y. F. \& Hines M. J. (1982). Initiation of walk and tiptoe of a planar nine link biped. Mathematical Biosciences, 61, 163-189.

Heemels W. P. M. H., Schumacher J. M. \& Weiland S. (1999). Linear complementarity systems. SIAM J. of Applied Mathematics., 60(4), 1234-1269.

Hirai K. (1997). Current and future perspective of Honda Humanoid Robots. Proc. IROS 1997, 500-508.

Hirai K., Hirose M. \& Kenada T. T. (1998). The development of Honda Humanoid Robot. Proc. of IEEE Int. Conf. on Robotics and Automation, Lewen, Belgium, May, 1321-1326.

Hodgins J., Raibert M. H. (1987). Biped gymnastics. Robotics Research: The 4th International Symposium, Bolles B. and Roth B. Eds, Cambridge.

$\mathrm{RR} \mathrm{n}^{\circ} 4290$ 
HONDA. U.S. Patents number : 5,432,417; 5,311,109; 5,355,064; 5,349,277; $5,426,586 ; 5,459,659 ; 5,357,433 ; 5,402,050 ; 5,252,901$.

Huang Q., Kaneko K., Yokoi K. Kajita S., Kotoku T., Koyachi N., Arai H., Imamura N., Komoriya K. \& Tanie K. (2000). Balance control of a biped robot combining off-line pattern with real-time modification. Proc. of the 2000 IEEE International Conference on Robotics and Automation.

Hurmuzlu Y. (1993). Dynamics of bipedal gait ; Part I - Objective functions and the contact event of a planar five link biped. Part II - Stability analysis of a planar five link biped. ASMEJournal of Applied Mechanics, 60(2), June, 331-344.

Hurmuzlu Y. \& Basdogan C. (1994), On the Measurement of Dynamic Stability of Human Locomotion., ASME Journal of Biomechanical Engineering, 116(1), $30-36$.

Hurmuzlu Y. \& Marghitu D. B. (1994). Rigid body collisions of planar kinematic chains with multiple contact points. Int. J. of Robotics Research, 13(1), February, 82-92.

Hurmuzlu Y. \& Moskowitz G. D. (1986). The role of impact in the stability of bipedal locomotion. Dynamics and Stability of Systems, 1(3), 217-234.

Hurmuzlu Y. \& Moskowitz G. D. (1987). Bipedal locomotion stabilized by impact and switching : I. Two and three dimensional, three elements models, II. Structural stability analysis of a four element bipedal locomotion model. Dynamics and Stability of Systems, 2(2), 73-112.

Hurmuzlu Y., Basdogan C. \& Stoianovici D. (1995), Kinematics and Dynamic Stability of the Locomotion of Polio Patients, ASME Journal of Biomechanical Engineering, 118, 405-411.

Hurmuzlu Y. (1998). An Energy Based Coefficient of Restitution for the Low Velocity Impacts of Slender Bars with Massive External Surfaces. ASMEJournal of Applied Mechanics, 65(4), 952-961.

IEEE Transactions on Automatic Control (1998). Special Issue on Hybrid Dynamical Systems, 43(4), April.

Igarashi E. \& Nogai T. (1992). Study of lower level adaptive walking in the sagittal plane by a biped locomotion robot. Advanced Robotics, 6(4), 441-459. 
Ivanov A. P. (1993). Stabilization of an impact oscillator near grazing incidence owing to resonance. J. of Sound and Vibrations, 162(3), 562-565.

Ivanov A. P. (1995). On multiple impacts. Prikl. Math. Mekh., 59(6), 930-946. Transl. in J. of Appl. Mech., 59(6), 887-902.

Jalics L., Hemami H. \& Clymer B. (1997). A control strategy for terrain adaptive bipedal locomotion". Autonomous Robots, 4, 243-257.

Jean M. (1993). Simulation numérique des problèmes de contact avec frottement. Matériaux et Techniques, 1-2-3, 22-32.

Kajita S., Yamaura T. \& Kobayashi A. (1992). Dynamic walking control of a biped robot along a potential energy conserving orbit. IEEE Transactions on Robotics and Automation, 8(4), 431-438.

Kajita S. \& Tani K. (1996). Experimental Study of Biped Dynamic Walking. IEEE Control Systems, 16, February, 13-19.

Kanzow C. (1996). Nonlinear complementarity as unconstrained optimization. Journal of Optimization Theory and Applications, 88(1), January, 139-155.

Katoh R. \& Mori M. (1984). Control Method of Biped Locomotion Giving Asymptotic Stability of Trajectory. Automatica, 20(4), 405-414.

Khosravi B., Yurkovich S. \& Hemami H. (1987). Control of a four link biped in a back somersault maneuver. IEEE Transactions on Systems, Man, and Cybernetics, 17(2), 303-325.

Kobrinskii A. E. (1965) Dynamics of mechanisms with elastic connections and impact systems, London, Ilife books Ltd.

Kozlov V. V. \& Treshchev D. V. (1991). Billiards. A genetic introduction to the dynamics of systems with impacts, Amer. Math. Soc., Providence, RI.

Kuhn H. W. \& Tucker A. W. (1951). Nonlinear programming. Proc. of the second Berkeley Symposium on Mathematical Statistics and Probability, Ed. J. Neyman, University of California, Press.

Kunze M. \& Neumann J. (1997) Linear Complementarity Systems and the Simulation of the Motion of Rigid. Body Systems Subject to Coulombs Friction. ZAMM-Z, Math. Mech, 77, 833-838.

$\mathrm{RR} \mathrm{n}^{\circ} 4290$ 
Kuo A. D. (1999). Stabilization of lateral motion in passive dynamic walking. Int. J. of Robotics Research, 18(9), 917-930, September.

Lapshin V. V. (1991). Motion control of a legged machine in the supportless phase of hopping. Int. J. of Robotics Research, 10(4), 327-337.

Lavrovskii E. K. (1979). Impact phenomena in problems of control of bipedal locomotion. izv. AN SSSR. Mekhanika Tverdogo Tela, 14(5), 41-47.

Lavrovskii E. K. (1980). Dynamics of bipedal locomotion at high velocity. $i z v . A N$ SSSR. Mekhanika Tverdogo Tela, 15(4), 50-58.

Lee T. T. \& Liao J. H. (1988). Trajectory planning and control of a 3-link biped robot. Proc. IEEE Conf. Robotics and Automation - New York, 820-823.

Lötstedt P. (1982). Mechanical systems of rigid bodies subject to unilateral constraints. SIAM J. Appl. Math., 42(2), 281-296, April.

Lötstedt P. (1984). Numerical simulation of time-dependent contact and friction problems in rigid body mechanics. SIAM J. Sci. Stat. Comput., 5(2), 370-393, June.

Mabrouk M. (1998). A unified variational model for the dynamics of perfect unilateral constraints". European J. of Mechanics A/Solids, 17(5), 819-848.

Marghitu D. B. \& Hurmuzlu Y. (1996). Nonlinear dynamics of an elastic rod with frictional impact. Nonlinear Dynamics, 10, 187-201.

Marghitu D. B. \& Hurmuzlu Y. (1995). Three Dimensional Rigid Body Collisions with Multiple Contact Points. ASME Journal of Applied Mechanics, 62, 725732 .

Masri S. F. \& Caughey T. K. (1966). On the stability of the impact damper. $A S M E$ J. of Applied Mechanics, 33, 586-592.

McClamroch N. H. \& Wang D. (1988). Feedback stabilization and tracking of constrained robots. IEEE Trans. on Automatic Control, 33(5), 419-426, May.

McGeer T. (1990). Passive Dynamic Walking. International Journal of Robotic Research, 9(2), 62-82.

Miura H. \& Shimoyama I. (1984). Dynamic walking of a biped. Int. J. of Robotics Research, 3(2), 60-74. 
Mochon S. \& McMahon T. A. (1980). Ballistic Walking. J. Biomech, 13, 49-57.

Mochon S. \& McMahon T. A. (1980). Ballistic Walking: An Improved Model. Math. Biosci., 52, 241-260.

Monteiro-Marques M. D. P. (1993). Differential Inclusions in Nonsmooth Mechanical Problems : Shocks and Dry Friction, Birkhauser, Boston PNLDE 9.

Moreau J. J. (1963). Les liaisons unilatérales et le principe de Gauss. C.R. Acad. Sciences Paris, 256, 871-874.

Moreau J. J. (1966). Quadratic programming in mechanics : dynamics of one sided constraints. J. SIAM Control, 4(1), 153-158.

Moreau J. J. (1985). Standard inelastic shocks and the dynamics of unilateral constraints". Unilateral Problems in Structural Analysis, G.Del Piero and F. Maceri (editors), CISM Courses and Lectures288, Springer-Verlag.

Moreau J. J. (1986). Dynamique de systèmes à liaisons unilatérales avec frottement sec éventuel ; essais numériques". Tech. note 85-1, LMGC, Université du Languedoc, Montpellier, France.

Moreau J. J., Panagiotopoulos P. D. (1988). Unilateral contact and dry friction in finite freedom dynamics. Nonsmooth mechanics and applications, CISM Courses and Lectures 302, Springer Verlag, 1-82.

Moreau J. J. (1994). Some numerical methods in multibody dynamics : application to granular materials. European J. of Mechanics A/Solids, 13(4), 93-114.

Neimark Y. I. (1995). Painleve paradoxes revisited. Mechanics of Solids, 30(1),1519. Izvestiya RAN, Mekhanika Tverdogo Tela, 1, 17-21, 1995.

Newby Jr. N. D. (1979). Linear collisions with harmonic oscillator forces : the inverse scattering problem. American Journal of Physics, 47, 161-165.

Novozhilov I. V. (1984). Control of three-dimensional motion of a bipedal walking robot. izv. AN SSSR. Mekhanika Tverdogo Tela, 19(4), 47-53.

Pang J. S. \& Trinkle J. C. (1996). Dynamic multi-rigid-body systems with concurrent distributed contacts. Submitted to Journal of Applied Mechanics.

$\operatorname{RR} n^{\circ} 4290$ 
Yildirim Hurmuzlu, Frank Génot, Bernard Brogliato

Paoli L. (1993). Analyse numérique de vibrations avec contraintes unilatérales, Ph.D. Thesis, Université Claude Bernard - Lyon 1, Laboratoire d'Analyse Numérique, France.

Paoli L. \& Schatzman M. (1993). Mouvement à nombre fini de degrés de liberté avec contraintes unilatérales : cas avec perte d'énergie". Mathematical Modelling and Numerical Analysis (Modélisation mathématique et analyse numérique), 27(6), 673-717.

Park J.H. \& Cho H.C. (2000). An on-line trajectory modifier for the base link of biped robots to enhance locomotion stability. Proc. of the 2000 IEEE International Conference on Robotics and Automation.

Parker T.S. \& Chua L.O. (1989). Practical numerical algorithms for chaotic systems., Springer-Verlag, New York.

Passino K. M., Michel A. N. \& Antsaklis P. J. (1994). Lyapunov stability of a class of discrete event systems. IEEE Transactions on Automatic Control, 39(2), 269-279, February.

Percivale D. (1985). Uniqueness in the elastic bounce problem. Journal of Differential Equations, 56, 206-215.

Percivale D. (1991). Uniqueness in the elastic bounce problem, II. Journal of Differential Equations, 90, 304-315.

Pérès J. (1953). Mécanique générale, Masson, Paris.

Perrin B., Chevallereau C. \& Formal'sky A. (1997). Control of a quadruped walking robot without feet for a gallop gait. IFAC Symposium on Robot Control (SYROCO'97), 3-5 September, Nantes, France.

Pfeiffer F. \& Glocker C. (1996). Multibody Dynamics with Unilateral Contacts, Wiley Series in Nonlinear Science.

Pratt G.A. \& Williamson M.M. (1995). Series elastic actuators. IEEE Conf. Intelligent Robots and Systems, 1, 399-406.

Pratt G.A. (2000). Legged robots at MIT: What's new since Raibert. IEEE Robotics and Automation Magazine, 7(3), september, 15-19. 
Pratt J., Chew C.-M., Torres A., Dilworth P., Pratt, G. (2001). An Intuitive Approach for Bipedal Locomotion. International Journal of Robotics Research, 20(2), 129-143.

Raibert M. H. (1986). Legged Robots That Balance, MIT Press, Cambridge, Mass.

Rubanovich E. M. \& Formal'sky A. M. (1981). Some problems of dynamics of multiple element systems associated with impact phenomena, II. izv. AN SSSR. Mekhanika Tverdogo Tela, 16(3), 125-133.

Rutkovskii S. V. (1985). Walking, skipping and running of a bipedal robot with allowance for impact. Mechanics of Solids, 20(5), 44-49.

Saunders J. B., Inman V. T. \& Eberhart H. D. (1953). The Major Determinants in Normal and Pathological Gait. J. Bone Jt. Surgery, 35(A), 543-558.

Schatzman M. (1978). A class of nonlinear differential equations of second order in time. Nonlinear Analysis, Theory, Methods and Applications, 2(3), 355-373.

Schatzman M. (1998). Uniqueness and continuous dependence on data for onedimensional impact problem. Math. Comput. Modeling, 28(4-8), 1-18.

Shaw J. \& Holmes P. (1983). A Periodically Forced Piecewise Linear Oscillator. Journal of Sound and Vibration, 90, 129-155.

Shaw J. \& Shaw S. (1989). The Onset of Chaos in a Two-Degree-of-Freedom Impacting System. ASME Journal of Applied Mechanics, 56, 168-174.

Shih C.L., Gruver W.A. \& Zhu Y. (1991). Fuzzy Logic Force Control for a Biped Robot. Proc. of the 1991 IEEE International Symposium on Intelligent Control, 269-273.

Shih C.L., Gruver W.A. \& Lee T.T. (1993). Inverse Kinematics and Inverse Dynamics for Control of a Biped Walking Machine. Journal of Robotic Systems, 10(4), $531-555$.

Shih C.L. \& Klein C. A. (1993). An adaptive gait for legged walking machines over rough terrain. IEEE Transactions on Systems, Man, and Cybernetics, 23(4), 1150-1155.

Slotine J. J. E. \& Li, W. (1991). Applied Nonlinear Control, Prentice Hall, New Jersey.

$\mathrm{RR} \mathrm{n}^{\circ} 4290$ 
Stewart D. E. (1997). Existence of solutions to rigid body dynamics and the Painlevé paradoxes. C.R. Acad. Sci. Paris, 325(1), 689-693.

Stewart D. E. (1998). Convergence of a time-stepping scheme for rigid body dynamics and resolution of Painlevé's paradoxes. Archives of Rational Mechanics Anal., 145, 215-260.

Stewart D. E. \& Trinkle J. C. (1996). An implicit time-stepping scheme for rigid body dynamics with inelastic collisions and Coulomb friction. International Journal of Numerical Methods in Engineering, 39(15), 2673-2691.

Stoianovici D. \& Hurmuzlu Y. (1996). A critical study of the applicability of rigid body collision theory. ASME Journal of Applied Mechanics, 63(2), 307-316.

Stronge W. J. (1990). Rigid body collision with friction. Proc. R. Soc. Lond. A, 431, 169-181.

Stronge W. J. (2000). Impact Mechanics, Cambridge University Press.

Takanishi A., Ishida M, Ymazaki Y \& Kato I. (1985). The realization of dynamic wlaking by the biped robot WL-10RD. Proc. of the Intl. Conf. on Adv. Robot., Tokyo, 459-466.

ten Dam A. A., Dwarshuis E. \& Willems J. C. (1997). The contact problem for linear continuous time dynamical system : a geometric approach. IEEE Trans. Automatic Control, 42(4), 458-472.

ten Dam A. A. (1997). Unilaterally constrained dynamical systems, PhD. thesis, Rijksuniversiteit Groningen, NL, http://www.ub.rug.nl/eldoc/dis/science/a.a.ten.dam/.

Todd D. J. (1985). Walking machines: An introduction to legged robots, Kogan Page, London.

Tornambe A. (1999). Modeling and control of the impact in mechanical systems. IEEE Trans. Automatic Control, 44(2), 294-309.

Trinkle J. C., Pang J. S., Sudarsky S. \& Lo G. (1995). On dynamic multi-rigid-body contact problems with Coulomb friction. Tech Report 95-003, Texas A \& M University, Departement of Computer Science. 
Tzitzouris J. A. \& Pang J. S. (2000). A time-stepping complementarity approach for frictionless systems of rigid bodies. Draft Technical Report, 600, The John Hopkins University, Mathematical Sciences Deptarment.

van der Schaft A. J. \& Schumacher J. M. (1998). Complementarity modeling of hybrid systems. IEEE Transactions on Automatic Control, special issue on Hybrid Systems, 43(4), 483-490, April.

van der Schaft A. J. \& Schumacher J. M. (1996). The complementary-slackness class of hybrid systems. Mathematics of Control, Signals and Systems, 9, 266-301.

Vukobratovic M. \& Juricic D. (1969). Contribution to the synthesis of biped gait. IEEE Trans. on Biomedical Engineering 16(1), 1-6.

Vukobratovic M. (1976). Walking robots and anthromopomorphic mechanics, MIR press, Russian Translation, Moskow.

Vukobratovic M., Borovac B., Surla D. \& Stokic D. (1990). Scientific Fundamentals of Robotics 7: Biped Locomotion, Springer-Verlag, New York.

Wang Y. \& Mason M. (1992). Two-dimensional rigid-body collisions with friction. ASME J. of Applied Mechanics, 59, 635-642.

Wieber P.B. (2000). Modélisation et commande d'un robot marcheur anthropomorphe. PhD Thesis, INRIA Rhône-Alpes, France, december.

Whittaker, E. T. (1904). A treatise on the analytical dynamics of particles and rigid bodies, Cambridge, UK, Cambridge Univ. Press.

Yang J. S. (1994). A control study of a kneeless biped locomotion system. Journal of the Franklin Institute, $\mathbf{3 3 1 b}(\mathbf{2})$, 125-143.

Zarrugh M. Y. (1976). Energy and Power in Human Walking, Ph.D Thesis, Univ. of California, Berkeley.

Zavala-Rio A. \& Brogliato B. (1999). On the control of a one degree-of-freedom juggling robot. Dynamics and Control, 9(7), 67-90.

Zheng Y. F. \& Hemami H. (1984). Impacts Effects of Biped Contact with the Environment. IEEE Transactions on Systems, Man, and Cybernetics, SMC-14(3), $437-443$.

RR $n^{\circ} 4290$ 
Zheng Y. F. (1989). Acceleration compensation for biped robot to reject external disturbances. IEEE Transactions on Systems, Man, and Cybernetics, 19(1), $74-84$.

Zheng Y.F. \& Sheng J. (1990). Gait synthesis for the SD-2 biped robot to climb sloping surface. IEEE Transactions on Robotics and Automation, 6(1), 86-96.

\section{A Continuous motion}

Walking can be seen as a sequence of sticking support phases. One of the main task the control law should achieve is to monitor the transitions between the successive contact modes, that is to guarantee the maintenance of some non-sliding ground contacts or to impose breaking at some others (transition from double to single support). Let us introduce the following sets of active constraints

$$
\mathcal{I}^{(p)}(t)=\left\{i \in\{1, \ldots, m\}, F_{i}(t)=0 \wedge \dot{F}_{i}(t)=0 \wedge \ldots \wedge F_{i}^{(p-1)}(t)=0\right\}
$$

where $p=1,2, \ldots$ is the order of the maximal derivation. Our aim is to derive necessary and sufficient conditions on the control law $u$ to maintain some sticking contacts $\mathcal{I}_{S} \subset \mathcal{I}^{(2)}(t)$ and to force breaking at the others $\mathcal{I}_{B}=\mathcal{I}^{(2)}(t) \backslash \mathcal{I}_{S}$.

$$
\forall i \in \mathcal{I}^{(2)}(t) \begin{cases}\ddot{F}_{i}=0, \quad \ddot{F}_{t, i}=0 & \text { if } i \in \mathcal{I}_{S} \\ \ddot{F}_{i}>0 & \text { otherwise }\end{cases}
$$

Let $r_{S}=\operatorname{card}\left(\mathcal{I}_{S}\right)$ and $r_{B}=\operatorname{card}\left(\mathcal{I}_{B}\right)$. In the sequel, if $X$ is a vector, $X_{S} \in \mathbb{R}^{r_{S}}$, resp. $X_{B} \in \mathbb{R}^{r_{B}}$, will denote the subvector relative to $\mathcal{I}_{S}$, resp. to $\mathcal{I}_{B}$. (59) can be rewritten as

$$
\begin{aligned}
\ddot{F}_{S} & =0 \\
\ddot{F}_{t, S} & =0 \\
\ddot{F}_{B} & >0
\end{aligned}
$$

A necessary and sufficient condition to guarantee (60) follows from the complementarity relation (10) between the normal acceleration $\ddot{F}_{S}$ and the normal forces $\lambda_{n, S}$

$$
\lambda_{n, S}>0
$$

In order to force stiction, Coulomb's dry friction law requires the contact forces to remain strictly inside the friction cone

$$
-\mu_{0, i} \lambda_{n, i}<\lambda_{t, i}<\mu_{0, i} \lambda_{n, i}, \quad i \in\{1, \ldots, m\}
$$


where $\mu_{0, i}$ is the friction coefficient at contact $i$. Introducing the diagonal matrix $\mu_{0, S}=\left(\operatorname{diag}\left[\mu_{0, i}\right], i \in\left\{1, \ldots, r_{S}\right\}\right)$, these conditions express

$$
\begin{aligned}
& \mu_{0, S} \lambda_{n, S}+\lambda_{t, S}>0 \\
& \mu_{0, S} \lambda_{n, S}-\lambda_{t, S}>0
\end{aligned}
$$

Using again the complementarity (10) between $\ddot{F}_{B}$ and $\lambda_{n, B}$, it follows from (62) that $\lambda_{n, B}=0$, and from Coulomb's friction, $\lambda_{t, B}=0$.

Let $\lambda_{S}^{T}=\left(\begin{array}{cc}\lambda_{n, S}^{T} & \lambda_{t, S}^{T}\end{array}\right)$ and $C_{S}(q)=\left(\begin{array}{cc}\nabla F_{S}(q) & G_{S}(q)\end{array}\right)$ where $G_{S}$ is such that $\dot{F}_{t, S}=G_{S}^{T} \dot{q}$. The dynamics (1) reduces to

$$
M(q) \ddot{q}=h(q, \dot{q}, u)+C_{S}(q) \lambda
$$

Let $\dot{k}_{S}^{T}=\left(\begin{array}{cc}\dot{F}_{S}^{T} & \dot{F}_{t, S}^{T}\end{array}\right)$

$$
\ddot{k}_{S}=C_{S}^{T} \ddot{q}+f_{S}(q, \dot{q})
$$

where $f_{S}^{T}=\left(\begin{array}{ll}f_{n, S}^{T} & f_{t, S}^{T}\end{array}\right)$. Inserting $\ddot{q}$ extracted from (66), (60) and (61) can be rewritten as

$$
C_{S}^{T} M^{-1} C_{S} \lambda_{S}=-C_{S}^{T} M^{-1} h-f_{S}
$$

In the sequel we will assume $C_{S}$ to be full rank. In this case $C_{S}^{T} M^{-1} C_{S}$ is positive symmetric definite, thus invertible

$$
\lambda_{S}=-\left(C_{S}^{T} M^{-1} C_{S}\right)^{-1}\left(C_{S}^{T} M^{-1} h_{S}+f_{S}\right)
$$

Introducing the orthogonal projections on $\nabla F_{S}^{\perp}$ et $G_{S}^{\perp}$ in the metric $M^{-1}$

$$
\begin{gathered}
P_{\nabla F_{S / M^{-1}}^{\perp}}=I d_{r_{S} \times r_{S}}-\nabla F_{S}\left(\nabla F_{S}^{T} M^{-1} \nabla F_{S}\right)^{-1} \nabla F_{S}^{T} M^{-1} \\
P_{G_{S / M^{-1}}^{\perp}}=I d_{r_{S} \times r_{S}}-G_{S}\left(G_{S}^{T} M^{-1} G_{S}\right)^{-1} G_{S}^{T} M^{-1}
\end{gathered}
$$

the expressions of the normal and tangential forces follow from (68)

$$
\begin{gathered}
\lambda_{n, S}(q, \dot{q}, u)=\left(\nabla F_{S}^{T} M^{-1} P_{G_{S / M^{-1}}^{\perp}} \nabla F_{S}\right)^{-1}\left(\nabla F_{S}^{T} M^{-1} G_{S}\left(G_{S}^{T} M^{-1} G_{S}\right)^{-1} f_{t, S}\right. \\
\left.-f_{n, S}-\nabla F_{S}^{T} M^{-1} P_{G_{S / M^{-1}}^{\perp}} h\right)
\end{gathered}
$$

$\mathrm{RR} \mathrm{n}^{\circ} 4290$ 
and

$$
\begin{gathered}
\lambda_{t, S}(q, \dot{q}, u)=\left(G_{S}^{T} M^{-1} P_{\nabla F_{S / M^{-1}}^{\perp}} G_{S}\right)^{-1}\left(G_{S}^{T} M^{-1} \nabla F_{S}\left(\nabla F_{S}^{T} M^{-1} \nabla F_{S}\right)^{-1} f_{n, S}\right. \\
\left.-f_{t, S}-G_{S}^{T} M^{-1} P_{\nabla F_{S / M^{-1}}^{\perp}} h\right)
\end{gathered}
$$

Finally

$$
\begin{aligned}
\ddot{F}_{B} & =\nabla F_{B}^{T} \ddot{q}+f_{n, B} \\
& =\nabla F_{B}^{T} M^{-1}\left(h+\nabla F_{S} \lambda_{n, S}+G_{S} \lambda_{t, S}\right)+f_{n, B}>0
\end{aligned}
$$

Notice that the expressions of the contact forces obtained in (69) and (70) are nonlinear in the state but linear in the control $u$. Thus it is possible to formulate the necessary and sufficient conditions (63), (64), (65) and (71) as

$$
A\left(q, \mu_{0, S}\right) u+B\left(q, \dot{q}, \mu_{0, S}\right)>0
$$

where $A \in \mathbb{R}^{\left(3 r_{S}+r_{B}\right) \times n}$ and $B \in \mathbb{R}^{3 r_{S}+r_{B}}$.

\section{B Impact-shaping}

Another problem arises at impact time. Sticking contacts at pre-impact can break or slide at post-impact. Due to the quasi-instantaneous feature of impact and to the limited bandwidth of the controller the only way in pratice to monitor the mode transition at impact is to elaborate a controller able to bring the system in a preimpact state compatible with the expected post-impact contact mode. Similarily to the continuous motion we may aim at expressing the constraints on $q\left(t_{k}\right)$ and $\dot{q}\left(t_{k}^{-}\right)$ such that some sticking contact $\mathcal{I}_{S} \subset \mathcal{I}^{(1)}\left(t_{k}^{-}\right)$at pre-impact will not slide nor break at post-impact and the others $\mathcal{I}_{B}=\mathcal{I}^{(1)}\left(t_{k}^{-}\right) \backslash \mathcal{I}_{S}$ will detach. Notice that $\mathcal{I}^{(1)}\left(t_{k}^{-}\right)$ contains not only all (sticking) contacts at pre-impact but also the impacting ones.

Since the impact outcome depends on the considered impact law, the constraints on the pre-impact state depend also strongly on the selected law. We opt for the one proposed by Pfeiffer and Glocker (Pfeiffer \& Glocker (1996)). It bases on Poisson's law, splitting the impact in a phase of compression (index $C$ ) lasting from $t_{A}=t_{k}^{-}$ to $t_{C}$, followed by a phase of expansion (index $E$ ) lasting from $t_{C}$ to $t_{E}=t_{k}^{+}$. We will do the classical assumption for biped robot that the multiple impact is plastic 
without tangential storage. In this case the expansion phase vanishes: $\dot{q}\left(t_{k}^{+}\right)=\dot{q}\left(t_{C}\right)$. We aim at realizing the following outcome

$$
\begin{aligned}
\dot{F}_{C, S} & =0 \\
\dot{F}_{t C, S} & =0 \\
\dot{F}_{C, B} & >0
\end{aligned}
$$

The end of the compression phase (and thus of the impact) is characterized in the following manner

- Since $\Lambda_{n, i} \geq 0, \forall t \in\left[t_{A}, t_{C}\right]$,

$$
\Lambda_{n C, i} \geq 0, \quad \forall i \in \mathcal{I}^{(1)}\left(t_{k}\right)
$$

- At the end of the compression phase, the approach between the two bodies involved is finished, i.e. negative values of the relative normal velocity are forbidden

$$
\dot{F}_{C, i} \geq 0, \quad \forall i \in \mathcal{I}^{(1)}\left(t_{k}\right)
$$

- If $\Lambda_{n C, i}=0$ then the corresponding constraint is useless and $\dot{F}_{C, i}$ can be any positive value. Otherwise the end of the compression is such that $\dot{F}_{C, i}=0$. Thus the following complementarity relation

$$
\Lambda_{n C, i} \dot{F}_{C, i}=0, \quad \forall i \in \mathcal{I}^{(1)}\left(t_{k}\right)
$$

The impact law in the tangential direction is given by

$$
\begin{aligned}
\left|\Lambda_{t C, i}\right|<\mu_{0, i} \Lambda_{n C, i} & \Rightarrow \dot{F}_{t C, i}=0 \\
\Lambda_{t C, i}=+\mu_{0, i} \Lambda_{n C, i} & \Rightarrow \quad \dot{F}_{t C, i} \leq 0 \\
\Lambda_{t C, i}=-\mu_{0, i} \Lambda_{n C, i} & \Rightarrow \quad \dot{F}_{t C, i} \geq 0
\end{aligned}
$$

It follows directly from the integration of Coulomb's law of friction over the impact phase. Nevertheless it doesn't imply that the contact force remains in the frictional cone during the whole compression process. So (79), (80) and (81) should be seen as an independent impact law in the tangential direction, see (Pfeiffer \& Glocker (1996), 110-116).

As for continuous motion, from (78), (75) leads to $\Lambda_{n C, B}=0$ and from the impact law in the tangential direction $\Lambda_{t C, B}=0$. The impact dynamics reduces to

$$
M \dot{q}_{C}=M \dot{q}_{A}+\nabla F_{S} \Lambda_{n C, S}+G_{S} \Lambda_{t C, S}
$$

RR $n^{\circ} 4290$ 
The experienced reader will have noticed that all computations made for the continuous motion (appendix A) can be transposed to new impact problem through the following rules of substitution

$$
\begin{cases}\ddot{F}_{S} & \longleftrightarrow \dot{F}_{C, S} \\ \ddot{F}_{t, S} & \longleftrightarrow \dot{F}_{t C, S} \\ \ddot{F}_{B} & \longleftrightarrow \dot{F}_{C, B} \\ \ddot{q} & \longleftrightarrow 0 \\ f_{n, S} & \longleftrightarrow 0 \\ f_{t, S} & \longleftrightarrow 0 \\ f_{n, B} & \longleftrightarrow M \dot{q}_{A} \\ h=T u-N & \longleftrightarrow \Lambda_{n C, S} \\ \lambda_{n, S} & \longleftrightarrow \Lambda_{t C, S}\end{cases}
$$

It follows directly (under the same assumption that $C_{S}$ is full rank)

$$
\begin{aligned}
\Lambda_{t C, S} & =-\left(G_{S}^{T} M^{-1} P_{\nabla F_{S / M^{-1}}^{\perp}} G_{S}\right)^{-1} G_{S}^{T} P_{\nabla F_{S / M}^{\perp-1}}^{T} \dot{q}\left(t_{k}^{-}\right) \\
& \triangleq \bar{\Lambda}_{t C, S}\left(q\left(t_{k}\right)\right) \dot{q}\left(t_{k}^{-}\right) \\
\Lambda_{n C, S} & =-\left(F_{S}^{T} M^{-1} P_{G_{S / M^{-1}}^{\perp}} F_{S}\right)^{-1} F_{S}^{T} P_{G_{S / M^{-1}}^{\perp}}^{T} \dot{q}\left(t_{k}^{-}\right) \\
& \triangleq \bar{\Lambda}_{n C, S}\left(q\left(t_{k}\right)\right) \dot{q}\left(t_{k}^{-}\right) \\
\dot{F}_{C, B} & =\nabla F_{B}^{T} M^{-1}\left(M+\nabla F_{S} \bar{\Lambda}_{n C, S}+G_{S} \bar{\Lambda}_{t C, S}\right) \dot{q}\left(t_{k}^{-}\right) \\
& \triangleq \overline{\dot{F}}_{C, B}\left(q\left(t_{k}\right)\right) \dot{q}\left(t_{k}^{-}\right)>0
\end{aligned}
$$

and the necessary and sufficient conditions

$$
\begin{aligned}
\bar{\Lambda}_{n C, S}\left(q\left(t_{k}\right)\right) \dot{q}\left(t_{k}^{-}\right) & >0 \\
\left(\mu_{0, S} \bar{\Lambda}_{n C, S}\left(q\left(t_{k}\right)\right)+\bar{\Lambda}_{t C, S}\left(q\left(t_{k}\right)\right)\right) \dot{q}\left(t_{k}^{-}\right) & >0 \\
\left(\mu_{0, S} \bar{\Lambda}_{n C, S}\left(q\left(t_{k}\right)\right)-\bar{\Lambda}_{t C, S}\left(q\left(t_{k}\right)\right)\right) \dot{q}\left(t_{k}^{-}\right) & >0 \\
\overline{\dot{F}}_{C, B}\left(q\left(t_{k}\right)\right) \dot{q}\left(t_{k}^{-}\right) & >0
\end{aligned}
$$

Similarily to the continuous motion, these conditions can be grouped as

$$
\bar{A}\left(q\left(t_{k}\right), \mu_{0, S}\right) \dot{q}\left(t_{k}^{-}\right)>0
$$

where $\bar{A} \in \mathbb{R}^{\left(3 r_{S}+r_{B}\right) \times n}$. 


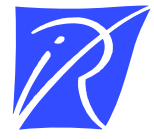

Unité de recherche INRIA Rhône-Alpes 655, avenue de l'Europe - 38330 Montbonnot-St-Martin (France)

Unité de recherche INRIA Lorraine : LORIA, Technopôle de Nancy-Brabois - Campus scientifique 615, rue du Jardin Botanique - BP 101 - 54602 Villers-lès-Nancy Cedex (France)

Unité de recherche INRIA Rennes : IRISA, Campus universitaire de Beaulieu - 35042 Rennes Cedex (France)

Unité de recherche INRIA Rocquencourt : Domaine de Voluceau - Rocquencourt - BP 105 - 78153 Le Chesnay Cedex (France)

Unité de recherche INRIA Sophia Antipolis : 2004, route des Lucioles - BP 93 - 06902 Sophia Antipolis Cedex (France)

INRIA - Domaine de Voluceau - Rocquencourt, BP 105 - 78153 Le Chesnay Cedex (France)

http://www.inria.fr

ISSN 0249-6399 